\title{
An analytically tractable model for competitive speciation
}

Pleuni S. Pennings (pennings@zi.biologie.uni-muenchen.de)

Michael Kopp (kopp@zi.biologie.uni-muenchen.de)

Géza Meszéna (geza.meszena@elte.hu)

Ulf Dieckmann (dieckmann@iiasa.ac.at)

Joachim Hermisson (hermisson@zi.biologie.uni-muenchen.de)

\section{Approved by}

Sten Nilsson

Director, IIASA

November 2008 


\section{IIASA STUDIES IN ADAPTIVE DYNAMICS No. 143}

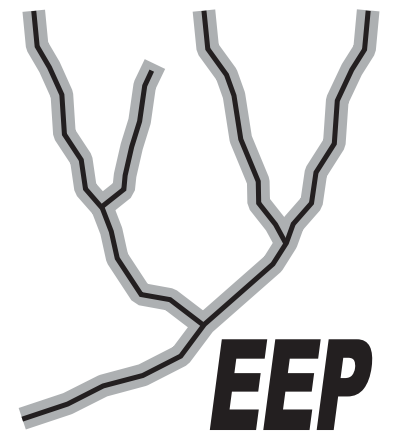

The Evolution and Ecology Program at IIASA fosters the development of new mathematical and conceptual techniques for understanding the evolution of complex adaptive systems.

Focusing on these long-term implications of adaptive processes in systems of limited growth, the Evolution and Ecology Program brings together scientists and institutions from around the world with IIASA acting as the central node.

Scientific progress within the network is collected in the IIASA Studies in Adaptive Dynamics series.
No. 1 Metz JAJ, Geritz SAH, Meszéna G, Jacobs FJA, van Heerwaarden JS: Adaptive Dynamics: A Geometrical Study of the Consequences of Nearly Faithful Reproduction. IIASA Working Paper WP-95-099 (1995). van Strien SJ, Verduyn Lunel SM (eds): Stochastic and Spatial Structures of Dynamical Systems, Proceedings of the Royal Dutch Academy of Science (KNAW Verhandelingen), North Holland, Amsterdam, pp. 183-231 (1996).

No. 2 Dieckmann U, Law R: The Dynamical Theory of Coevolution: A Derivation from Stochastic Ecological Processes. IIASA Working Paper WP-96-001 (1996). Journal of Mathematical Biology 34:579-612 (1996).

No. 3 Dieckmann U, Marrow P, Law R: Evolutionary Cycling of Predator-PreyInteractions: Population Dynamics and the Red Queen. IIASA Preprint (1995). Journal of Theoretical Biology 176:91-102 (1995).

No. 4 Marrow P, Dieckmann U, Law R: Evolutionary Dynamics of Predator-Prey Systems: An Ecological Perspective. IIASA Working Paper WP-96-002 (1996). Journal of Mathematical Biology 34:556-578 (1996).

No. 5 Law R, Marrow P, Dieckmann U: On Evolution under Asymmetric Competition. IIASA Working Paper WP-96-003 (1996). Evolutionary Ecology 11:485-501 (1997).

No. 6 Metz JAJ, Mylius SD, Diekmann O: When Does Evolution Optimize? On the Relation Between Types of Density Dependence and Evolutionarily Stable Life History Parameters. IIASA Working Paper WP-96-004 (1996).

No. 7 Ferrière R, Gatto M: Lyapunov Exponents and the Mathematics of Invasion in Oscillatory or Chaotic Populations. Theoretical Population Biology 48:126-171 (1995).

No. 8 Ferrière R, Fox GA: Chaos and Evolution. IIASA Preprint (1996). Trends in Ecology and Evolution 10:480485 (1995).

No. 9 Ferrière R, Michod RE: The Evolution of Cooperation in Spatially Heterogeneous Populations. IIASA Working Paper WP-96-029 (1996). The American Naturalist 147:692717 (1996).

No. 10 van Dooren TJM, Metz JAJ: Delayed Maturation in Temporally Structured Populations with Non-Equilibrium Dynamics. IIASA Working Paper WP-96-070 (1996). Journal of Evolutionary Biology 11:41-62 (1998).
No. 11 Geritz SAH, Metz JAJ, Kisdi É, Meszéna G: The Dynamics of Adaptation and Evolutionary Branching. IIASA Working Paper WP-96-077 (1996). Physical Review Letters 78:2024-2027 (1997).

No. 12 Geritz SAH, Kisdi É, Meszéna G, Metz JAJ: Evolutionary Singular Strategies and the Adaptive Growth and Branching of the Evolutionary Tree. IIASA Working Paper WP-96-114 (1996). Evolutionary Ecology 12:35-57 (1998).

No. 13 Heino M, Metz JAJ, Kaitala V: Evolution of Mixed Maturation Strategies in Semelparous Life-Histories: The Crucial Role of Dimensionality of Feedback Environment. IIASA Working Paper WP-96-126 (1996). Philosophical Transactions of the Royal Society of London Series B 352:1647-1655 (1997).

No. 14 Dieckmann U: Can Adaptive Dynamics Invade? IIASA Working Paper WP-96-152 (1996). Trends in Ecology and Evolution 12:128-131 (1997).

No. 15 Meszéna G, Czibula I, Geritz SAH: Adaptive Dynamics in a 2-Patch Environment: A Simple Model for Allopatric and Parapatric Speciation. IIASA Interim Report IR-97-001 (1997). Journal of Biological Systems 5:265-284 (1997).

No. 16 Heino M, Metz JAJ, Kaitala V: The Enigma of Frequency-Dependent Selection. IIASA Interim Report IR97-061 (1997). Trends in Ecology and Evolution 13:367-370 (1998).

No. 17 Heino M: Management of Evolving Fish Stocks. IIASA Interim Report IR-97-062 (1997). Canadian Journal of Fisheries and Aquatic Sciences 55:1971-1982 (1998).

No. 18 Heino M: Evolution of Mixed Reproductive Strategies in Simple Life-History Models. IIASA Interim Report IR-97063 (1997).

No. 19 Geritz SAH, van der Meijden E, Metz JAJ: Evolutionary Dynamics of Seed Size and Seedling Competitive Ability. IIASA Interim Report IR-97-071 (1997). Theoretical Population Biology 55:324-343 (1999).

No. 20 Galis F, Metz JAJ: Why Are There So Many Cichlid Species? On the Interplay of Speciation and Adaptive Radiation. IIASA Interim Report IR-97-072 (1997). Trends in Ecology and Evolution 13:1-2 (1998). 
No. 21 Boerlijst MC, Nowak MA, Sigmund K: Equal Pay for all Prisoners/ The Logic of Contrition. IIASA Interim Report IR-97-073 (1997). American Mathematical Society Monthly 104:303-307 (1997). Journal of Theoretical Biology 185:281-293 (1997).

No. 22 Law R, Dieckmann U: Symbiosis Without Mutualism and the Merger of Lineages in Evolution. IIASA Interim Report IR-97-074 (1997). Proceedings of the Royal Society of London Series B 265:1245-1253 (1998).

No. 23 Klinkhamer PGL, de Jong TJ, Metz JAJ: Sex and Size in Cosexual Plants. IIASA Interim Report IR-97-078 (1997). Trends in Ecology and Evolution 12:260-265 (1997).

No. 24 Fontana W, Schuster P: Shaping Space: The Possible and the Attainable in RNA Genotype-Phenotype Mapping. IIASA Interim Report IR-98-004 (1998). Journal of Theoretical Biology 194:491-515 (1998).

No. 25 Kisdi É, Geritz SAH: Adaptive Dynamics in Allele Space: Evolution of Genetic Polymorphism by Small Mutations in a Heterogeneous Environment. IIASA Interim Report IR-98-038 (1998). Evolution 53:993-1008 (1999).

No. 26 Fontana W, Schuster P: Continuity in Evolution: On the Nature of Transitions. IIASA Interim Report IR-98-039 (1998). Science 280:1451-1455 (1998).

No. 27 Nowak MA, Sigmund K: Evolution of Indirect Reciprocity by Image Scoring/ The Dynamics of Indirect Reciprocity. IIASA Interim Report IR-98-040 (1998). Nature 393:573-577 (1998). Journal of Theoretical Biology 194:561574 (1998).

No. 28 Kisdi É: Evolutionary Branching Under Asymmetric Competition. IIASA Interim Report IR-98-045 (1998). Journal of Theoretical Biology 197:149-162 (1999).

No. 29 Berger U: Best Response Adaptation for Role Games. IIASA Interim Report IR-98-086 (1998).

No. 30 van Dooren TJM: The Evolutionary Ecology of Dominance-Recessivity. IIASA Interim Report IR-98-096 (1998). Journal of Theoretical Biology 198:519-532 (1999).

No. 31 Dieckmann U, O'Hara B, Weisser W: The Evolutionary Ecology of Dispersal. IIASA Interim Report IR-98-108 (1998). Trends in Ecology and Evolution 14:88-90 (1999).

No. 32 Sigmund K: Complex Adaptive Systems and the Evolution of Reciprocation. IIASA Interim Report IR-98-100 (1998). Ecosystems 1:444-448 (1998).

No. 33 Posch M, Pichler A, Sigmund K: The Efficiency of Adapting Aspiration Levels. IIASA Interim Report IR-98103 (1998). Proceedings of the Royal Society London Series B 266:1427-1435 (1999).

No. 34 Mathias A, Kisdi É: Evolutionary Branching and Coexistence of Germination Strategies. IIASA Interim Report IR-99-014 (1999).

No. 35 Dieckmann U, Doebeli M: On the Origin of Species by Sympatric Speciation. IIASA Interim Report IR-99-013 (1999). Nature 400:354-357 (1999).

No. 36 Metz JAJ, Gyllenberg M: How Should We Define Fitness in Structured Metapopulation Models? Including an Application to the Calculation of Evolutionarily Stable Dispersal Strategies. IIASA Interim Report IR-99-019 (1999). Proceedings of the Royal Society of London Series B 268:499508 (2001)
No. 37 Gyllenberg M, Metz JAJ: On Fitness in Structured Metapopulations. IIASA Interim Report IR-99-037 (1999). Journal of Mathematical Biology 43:545-560 (2001).

No. 38 Meszéna G, Metz JAJ: Species Diversity and Population Regulation: The Importance of Environmental Feedback Dimensionality. IIASA Interim Report IR-99-045 (1999).

No. 39 Kisdi É, Geritz SAH: Evolutionary Branching and Sympatric Speciation in Diploid Populations. IIASA Interim Report IR-99-048 (1999).

No. 40 Ylikarjula J, Heino M, Dieckmann U: Ecology and Adaptation of Stunted Growth in Fish. IIASA Interim Report IR-99-050 (1999). Evolutionary Ecology 13:433-453 (1999).

No. 41 Nowak MA, Sigmund K: Games on Grids. IIASA Interim Report IR-99-038 (1999). Dieckmann U, Law R, Metz JAJ (eds): The Geometry of Ecological Interactions: Simplifying Spatial Complexity, Cambridge University Press, Cambridge, UK, pp. 135-150 (2000).

No. 42 Ferrière R, Michod RE: Wave Patterns in Spatial Games and the Evolution of Cooperation. IIASA Interim Report IR-99-041 (1999). Dieckmann U, Law R, Metz JAJ (eds): The Geometry of Ecological Interactions: Simplifying Spatial Complexity, Cambridge University Press, Cambridge, UK, pp. 318-332 (2000).

No. 43 Kisdi É, Jacobs FJA, Geritz SAH: Red Queen Evolution by Cycles of Evolutionary Branching and Extinction. IIASA Interim Report IR-00-030 (2000). Selection 2:161$176(2001)$.

No. 44 Meszéna G, Kisdi É, Dieckmann U, Geritz SAH, Metz JAJ: Evolutionary Optimisation Models and Matrix Games in the Unified Perspective of Adaptive Dynamics. IIASA Interim Report IR-00-039 (2000). Selection 2:193-210 (2001).

No. 45 Parvinen K, Dieckmann U, Gyllenberg M, Metz JAJ: Evolution of Dispersal in Metapopulations with Local Density Dependence and Demographic Stochasticity. IIASA Interim Report IR-00-035 (2000). Journal of Evolutionary Biology 16:143-153 (2003).

No. 46 Doebeli M, Dieckmann U: Evolutionary Branching and Sympatric Speciation Caused by Different Types of Ecological Interactions. IIASA Interim Report IR-00-040 (2000). The American Naturalist 156:S77-S101 (2000).

No. 47 Heino M, Hanski I: Evolution of Migration Rate in a Spatially Realistic Metapopulation Model. IIASA Interim Report IR-00-044 (2000). The American Naturalist 157:495$511(2001)$.

No. 48 Gyllenberg M, Parvinen K, Dieckmann U: Evolutionary Suicide and Evolution of Dispersal in Structured Metapopulations. IIASA Interim Report IR-00-056 (2000). Journal of Mathematical Biology 45:79-105 (2002).

No. 49 van Dooren TJM: The Evolutionary Dynamics of Direct Phenotypic Overdominance: Emergence Possible, Loss Probable. IIASA Interim Report IR-00-048 (2000). Evolution 54:1899-1914 (2000).

No. 50 Nowak MA, Page KM, Sigmund K: Fairness Versus Reason in the Ultimatum Game. IIASA Interim Report IR00-57 (2000). Science 289:1773-1775 (2000).

No. 51 de Feo O, Ferrière R: Bifurcation Analysis of Population Invasion: On-Off Intermittency and Basin Riddling. IIASA Interim Report IR-00-074 (2000). International Journal of Bifurcation and Chaos 10:443-452 (2000). 
No. 52 Heino M, Laaka-Lindberg S: Clonal Dynamics and Evolution of Dormancy in the Leafy Hepatic Lophozia Silvicola. IIASA Interim Report IR-01-018 (2001). Oikos 94:525-532 (2001).

No. 53 Sigmund K, Hauert C, Nowak MA: Reward and Punishment in Minigames. IIASA Interim Report IR-01-031 (2001). Proceedings of the National Academy of Sciences of the USA 98:10757-10762 (2001).

No. 54 Hauert C, De Monte S, Sigmund K, Hofbauer J: Oscillations in Optional Public Good Games. IIASA Interim Report IR-01-036 (2001).

No. 55 Ferrière R, Le Galliard J: Invasion Fitness and Adaptive Dynamics in Spatial Population Models. IIASA Interim Report IR-01-043 (2001). Clobert J, Dhondt A, Danchin E, Nichols J (eds): Dispersal, Oxford University Press, pp. 57-79 (2001).

No. 56 de Mazancourt C, Loreau M, Dieckmann U: Can the Evolution of Plant Defense Lead to Plant-Herbivore Mutualism? IIASA Interim Report IR-01-053 (2001). The American Naturalist 158:109-123 (2001).

No. 57 Claessen D, Dieckmann U: Ontogenetic Niche Shifts and Evolutionary Branching in Size-Structured Populations. IIASA Interim Report IR-01-056 (2001). Evolutionary Ecology Research 4:189-217 (2002).

No. 58 Brandt H: Correlation Analysis of Fitness Landscapes. IIASA Interim Report IR-01-058 (2001).

No. 59 Dieckmann U: Adaptive Dynamics of Pathogen-Host Interacations. IIASA Interim Report IR-02-007 (2002). Dieckmann U, Metz JAJ, Sabelis MW, Sigmund K (eds): Adaptive Dynamics of Infectious Diseases: In Pursuit of Virulence Management, Cambridge University Press, Cambridge, UK, pp. 39-59 (2002).

No. 60 Nowak MA, Sigmund K: Super- and Coinfection: The Two Extremes. IIASA Interim Report IR-02-008 (2002). Dieckmann U, Metz JAJ, Sabelis MW, Sigmund K (eds): Adaptive Dynamics of Infectious Diseases: In Pursuit of Virulence Management, Cambridge University Press, Cambridge, UK, pp. 124-137 (2002).

No. 61 Sabelis MW, Metz JAJ: Evolution Management: Taking Stock - Relating Theory to Experiment. IIASA Interim Report IR-02-009 (2002). Dieckmann U, Metz JAJ, Sabelis MW, Sigmund K (eds): Adaptive Dynamics of Infectious Diseases: In Pursuit of Virulence Management, Cambridge University Press, Cambridge, UK, pp. 379-398 (2002).

No. 62 Cheptou P, Dieckmann U: The Evolution of SelfFertilization in Density-Regulated Populations . IIASA Interim Report IR-02-024 (2002). Proceedings of the Royal Society of London Series B 269:1177-1186(2002).

No. 63 Bürger R: Additive Genetic Variation Under Intraspecific Competition and Stabilizing Selection: A Two-Locus Study. IIASA Interim Report IR-02-013 (2002). Theoretical Population Biology 61:197-213 (2002).

No. 64 Hauert C, De Monte S, Hofbauer J, Sigmund K: Volunteering as Red Queen Mechanism for Co-operation in Public Goods Games. IIASA Interim Report IR-02-041 (2002). Science 296:1129-1132 (2002).

No. 65 Dercole F, Ferrière R, Rinaldi S: Ecological Bistability and Evolutionary Reversals under Asymmetrical Competition. IIASA Interim Report IR-02-053 (2002). Evolution 56:1081-1090 (2002).
No. 66 Dercole F, Rinaldi S: Evolution of Cannibalistic Traits: Scenarios Derived from Adaptive Dynamics. IIASA Interim Report IR-02-054 (2002). Theoretical Population Biology 62:365-374 (2002).

No. 67 Bürger R, Gimelfarb A: Fluctuating Environments and the Role of Mutation in Maintaining Quantitative Genetic Variation. IIASA Interim Report IR-02-058 (2002). Genetical Research 80:31-46 (2002).

No. 68 Bürger R: On a Genetic Model of Intraspecific Competition and Stabilizing Selection. IIASA Interim Report IR02-062 (2002). Amer. Natur. 160:661-682 (2002).

No. 69 Doebeli M, Dieckmann U: Speciation Along Environmental Gradients. IIASA Interim Report IR-02-079 (2002). Nature 421:259-264 (2003).

No. 70 Dercole F, Irisson J, Rinaldi S: Bifurcation Analysis of a Prey-Predator Coevolution Model. IIASA Interim Report IR-02-078 (2002). SIAM Journal on Applied Mathematics 63:1378-1391 (2003).

No. 71 Le Galliard J, Ferrière R, Dieckmann U: The Adaptive Dynamics of Altruism in Spatially Heterogeneous Populations. IIASA Interim Report IR-03-006 (2003). Evolution 57:1-17 (2003).

No. 72 Taborsky B, Dieckmann U, Heino M: Unexpected Discontinuities in Life-History Evolution under SizeDependent Mortality. IIASA Interim Report IR-03-004 (2003). Proceedings of the Royal Society of London Series B 270:713-721 (2003).

No. 73 Gardmark A, Dieckmann U, Lundberg P: LifeHistory Evolution in Harvested Populations: The Role of Natural Predation. IIASA Interim Report IR-03-008 (2003). Evolutionary Ecology Research 5:239-257 (2003).

No. 74 Mizera F, Meszéna G: Spatial Niche Packing, Character Displacement and Adaptive Speciation Along an Environmental Gradient. IIASA Interim Report IR-03-062 (2003). Evolutionary Ecology Research 5:363-382 (2003).

No. 75 Dercole F: Remarks on Branching-Extinction Evolutionary Cycles. IIASA Interim Report IR-03-077 (2003). Journal of Mathematical Biology 47:569-580 (2003).

No. 76 Hofbauer J, Sigmund K: Evolutionary Game Dynamics. IIASA Interim Report IR-03-078 (2003). Bulletin of the American Mathematical Society 40:479-519 (2003).

No. 77 Ernande B, Dieckmann U, Heino M: Adaptive Changes in Harvested Populations: Plasticity and Evolution of Age and Size at Maturation. IIASA Interim Report IR03-058 (2003). Proceedings of the Royal Society of London Series B-Biological Sciences 271:415-423 (2004).

No. 78 Hanski I, Heino M: Metapopulation-Level Adaptation of Insect Host Plant Preference and Extinction-Colonization Dynamics in Heterogeneous Landscapes. IIASA Interim Report IR-03-028 (2003). Theoretical Population Biology 63:309-338 (2003).

No. 79 van Doorn G, Dieckmann U, Weissing FJ: Sympatric Speciation by Sexual Selection: A Critical Re-Evaluation. IIASA Interim Report IR-04-003 (2004). American Naturalist 163:709-725 (2004).

No. 80 Egas M, Dieckmann U, Sabelis MW: Evolution Restricts the Coexistence of Specialists and Generalists - the Role of Trade-off Structure. IIASA Interim Report IR-04-004 (2004). American Naturalist 163:518-531 (2004). 
No. 81 Ernande B, Dieckmann U: The Evolution of Phenotypic Plasticity in Spatially Structured Environments: Implications of Intraspecific Competition, Plasticity Costs, and Environmental Characteristics. IIASA Interim Report IR-04-006 (2004). Journal of Evolutionary Biology 17:613-628 (2004).

No. 82 Cressman R, Hofbauer J: Measure Dynamics on a One-Dimensional Continuous Trait Space: Theoretical Foundations for Adaptive Dynamics. IIASA Interim Report IR04-016 (2004).

No. 83 Cressman R: Dynamic Stability of the Replicator Equation with Continuous Strategy Space. IIASA Interim Report IR-04-017 (2004).

No. 84 Ravigné V, Olivieri I, Dieckmann U: Implications of Habitat Choice for Protected Polymorphisms. IIASA Interim Report IR-04-005 (2004). Evolutionary Ecology Research 6:125-145 (2004).

No. 85 Nowak MA, Sigmund K: Evolutionary Dynamics of Biological Games. IIASA Interim Report IR-04-013 (2004). Science 303:793-799 (2004).

No. 86 Vukics A, Asbóth J, Meszéna G: Speciation in Multidimensional Evolutionary Space. IIASA Interim Report IR-04-028 (2004). Physical Review 68:041-903 (2003).

No. 87 de Mazancourt C, Dieckmann U: Trade-off Geometries and Frequency-dependent Selection. IIASA Interim Report IR-04-039 (2004). American Naturalist 164:765-778 (2004).

No. 88 Cadet CR, Metz JAJ, Klinkhamer PGL: Size and the Not-So-Single Sex: Disentangling the Effects of Size on Sex Allocation. IIASA Interim Report IR-04-084 (2004). American Naturalist 164:779-792 (2004).

No. 89 Rueffler C, van Dooren TJM, Metz JAJ: Adaptive Walks on Changing Landscapes: Levins' Approach Extended. IIASA Interim Report IR-04-083 (2004). Theoretical Population Biology 65:165-178 (2004).

No. 90 de Mazancourt C, Loreau M, Dieckmann U: Understanding Mutualism When There is Adaptation to the Partner. IIASA Interim Report IR-05-016 (2005). Journal of Ecology 93:305-314 (2005).

No. 91 Dieckmann U, Doebeli M: Pluralism in Evolutionary Theory. IIASA Interim Report IR-05-017 (2005). Journal of Evolutionary Biology 18:1209-1213 (2005).

No. 92 Doebeli M, Dieckmann U, Metz JAJ, Tautz D: What We Have Also Learned: Adaptive Speciation is Theoretically Plausible. IIASA Interim Report IR-05-018 (2005). Evolution 59:691-695 (2005).

No. 93 Egas M, Sabelis MW, Dieckmann U: Evolution of Specialization and Ecological Character Displacement of Herbivores Along a Gradient of Plant Quality. IIASA Interim Report IR-05-019 (2005). Evolution 59:507-520 (2005).

No. 94 Le Galliard J, Ferrière R, Dieckmann U: Adaptive Evolution of Social Traits: Origin, Trajectories, and Correlations of Altruism and Mobility. IIASA Interim Report IR05-020 (2005). American Naturalist 165:206-224 (2005).

No. 95 Doebeli M, Dieckmann U: Adaptive Dynamics as a Mathematical Tool for Studying the Ecology of Speciation Processes. IIASA Interim Report IR-05-022 (2005). Journal of Evolutionary Biology 18:1194-1200 (2005).

No. 96 Brandt H, Sigmund K: The Logic of Reprobation: Assessment and Action Rules for Indirect Reciprocity. IIASA Interim Report IR-04-085 (2004). Journal of Theoretical Biology 231:475-486 (2004).
No. 97 Hauert C, Haiden N, Sigmund K: The Dynamics of Public Goods. IIASA Interim Report IR-04-086 (2004). Discrete and Continuous Dynamical Systems - Series B 4:575587 (2004).

No. 98 Meszéna G, Gyllenberg M, Jacobs FJA, Metz JAJ: Link Between Population Dynamics and Dynamics of Darwinian Evolution. IIASA Interim Report IR-05-026 (2005). Physical Review Letters 95:Article 078105 (2005).

No. 99 Meszéna G: Adaptive Dynamics: The Continuity Argument. IIASA Interim Report IR-05-032 (2005).

No. 100 Brännström NA, Dieckmann U: Evolutionary Dynamics of Altruism and Cheating Among Social Amoebas. IIASA Interim Report IR-05-039 (2005). Proceedings of the Royal Society London Series B 272:1609-1616 (2005).

No. 101 Meszéna G, Gyllenberg M, Pasztor L, Metz JAJ: Competitive Exclusion and Limiting Similarity: A Unified Theory. IIASA Interim Report IR-05-040 (2005).

No. 102 Szabo P, Meszéna G: Limiting Similarity Revisited. IIASA Interim Report IR-05-050 (2005).

No. 103 Krakauer DC, Sasaki A: The Greater than Two-Fold Cost of Integration for Retroviruses. IIASA Interim Report IR-05-069 (2005).

No. 104 Metz JAJ: Eight Personal Rules for Doing Science. IIASA Interim Report IR-05-073 (2005). Journal of Evolutionary Biology 18:1178-1181 (2005).

No. 105 Beltman JB, Metz JAJ: Speciation: More Likely Through a Genetic or Through a Learned Habitat Preference? IIASA Interim Report IR-05-072 (2005). Proceedings of the Royal Society of London Series B 272:1455-1463 (2005).

No. 106 Durinx M, Metz JAJ: Multi-type Branching Processes and Adaptive Dynamics of Structured Populations. IIASA Interim Report IR-05-074 (2005). Haccou P, Jager P, Vatutin V (eds): Branching Processes: Variation, Growth and Extinction of Populations, Cambridge University Press, Cambridge, UK, pp. 266-278 (2005).

No. 107 Brandt H, Sigmund K: The Good, the Bad and the Discriminator - Errors in Direct and Indirect Reciprocity. IIASA Interim Report IR-05-070 (2005). Journal of Theoretical Biology 239:183-194 (2006).

No. 108 Brandt H, Hauert C, Sigmund K: Punishing and Abstaining for Public Goods. IIASA Interim Report IR-05-071 (2005). Proceedings of the National Academy of Sciences of the United States of America 103:495-497 (2006).

No. 109 Ohtsuki A, Sasaki A: Epidemiology and DiseaseControl Under Gene-for-Gene Plant-Pathogen Interaction. IIASA Interim Report IR-05-068 (2005).

No. 110 Brandt H, Sigmund K: Indirect Reciprocity, ImageScoring, and Moral Hazard. IIASA Interim Report IR-05078 (2005). Proceedings of the National Academy of Sciences of the United States of America 102:2666-2670 (2005).

No. 111 Nowak MA, Sigmund K: Evolution of Indirect Reciprocity. IIASA Interim Report IR-05-079 (2005). Nature 437:1292-1298 (2005).

No. 112 Kamo M, Sasaki A: Evolution Towards Multi-Year Periodicity in Epidemics. IIASA Interim Report IR-05-080 (2005). Ecology Letters 8:378-385 (2005). 
No. 113 Dercole F, Ferrière R, Gragnani A, Rinaldi S: Coevolution of Slow-fast Populations: Evolutionary Sliding, Evolutionoary Pseudo-equilibria, and Complex Red Queen Dynamics. IIASA Interim Report IR-06-006 (2006). Proceedings of the Royal Society B 273:983-990 (2006).

No. 114 Dercole F: Border Collision Bifurcations in the Evolution of Mutualistic Interactions. IIASA Interim Report IR-05-083 (2005). International Journal of Bifurcation and Chaos 15:2179-2190 (2005).

No. 115 Dieckmann U, Heino M, Parvinen K: The Adaptive Dynamics of Function-Valued Traits. IIASA Interim Report IR-06-036 (2006). Journal of Theoretical Biology 241:370389 (2006)

No. 116 Dieckmann U, Metz JAJ: Surprising Evolutionary Predictions from Enhanced Ecological Realism. IIASA Interim Report IR-06-037 (2006). Theoretical Population Biology 69:263-281 (2006).

No. 117 Dieckmann U, Brännström NA, HilleRisLambers R, Ito H: The Adaptive Dynamics of Community Structure. IIASA Interim Report IR-06-038 (2006). Takeuchi Y, Iwasa Y, Sato K (eds): Mathematics for Ecology and Environmental Sciences, Springer, Berlin Heidelberg, pp. 145-177 (2007).

No. 118 Gardmark A, Dieckmann U: Disparate Maturation Adaptations to Size-dependent Mortality. IIASA Interim Report IR-06-039 (2006). Proceedings of the Royal Society London Series B 273:2185-2192(2006).

No. 119 van Doorn G, Dieckmann U: The Long-term Evolution of Multi-locus Traits Under Frequency-dependent Disruptive Selection. IIASA Interim Report IR-06-041 (2006). Evolution 60:2226-2238 (2006).

No. 120 Doebeli M, Blok HJ, Leimar O, Dieckmann U: Multimodal Pattern Formation in Phenotype Distributions of Sexual Populations. IIASA Interim Report IR-06-046 (2006). Proceedings of the Royal Society London Series B 274:347357 (2007).

No. 121 Dunlop ES, Shuter BJ, Dieckmann U: The Demographic and Evolutionary Consequences of Selective Mortality: Predictions from an Eco-genetic Model of the Smallmouth Bass. IIASA Interim Report IR-06-060 (2006). Transactions of the American Fisheries Society 136:749-765 (2007).

No. 122 Metz JAJ: Fitness. IIASA Interim Report IR-06061 (2006).

No. 123 Brandt H, Ohtsuki H, Iwasa Y, Sigmund K: A Survey on Indirect Reciprocity. IIASA Interim Report IR-06-065 (2006). Takeuchi Y, Iwasa Y, Sato K (eds): Mathematics for Ecology and Environmental Sciences, Springer, Berlin Heidelberg, pp. 21-51 (2007).

No. 124 Dercole F, Loiacono D, Rinaldi S: Synchronization in Ecological Networks: A Byproduct of Darwinian Evolution? IIASA Interim Report IR-06-068 (2006). International Journal of Bifurcation and Chaos 7:2435-2446 (2007).

No. 125 Dercole F, Dieckmann U, Obersteiner M, Rinaldi S: Adaptive Dynamics and Technological Change. IIASA Interim Report IR-06-070 (2006). Technovation 28:335-348 (2008).

No. 126 Rueffler C, van Dooren TJM, Metz JAJ: The Evolution of Resource Specialization Through FrequencyDependent and Frequency-Independent Mechanisms. IIASA Interim Report IR-06-073 (2006). American Naturalist 167:81-93 (2006).

No. 127 Rueffler C, Egas M, Metz JAJ: Evolutionary Predictions Should be Based on Individual Traits. IIASA Interim Report IR-06-074 (2006). American Naturalist 168:148-162 (2006).
No. 128 Kamo M, Sasaki A, Boots M: The Role of Trade-Off Shapes in the Evolution of Virulence in Spatial Host-Parasite Interactions: An Approximate Analytical Approach . IIASA Interim Report IR-06-075 (2006)

No. 129 Boots M, Kamo M, Sasaki A: The Implications of Spatial Structure Within Populations to the Evolution of Parasites. IIASA Interim Report IR-06-078 (2006).

No. 130 Andreasen V, Sasaki A: Shaping the Phylogenetic Tree of Influenza by Cross-Immunity. IIASA Interim Report IR-06-079 (2006).

No. 131 Rueffler C, van Dooren TJM, Metz JAJ: The Interplay Between Behavior and Morphology in the Evolutionary Dynamics of Resource Specialization. IIASA Interim Report IR-06-082 (2006). American Naturalist 169:E34-E52 (2007).

No. 132 Rueffler C, van Dooren TJM, Metz JAJ: The Evolution of Simple Life-Histories: Steps Towards a Classification. IIASA Interim Report IR-06-083 (2006).

No. 133 Durinx M, Metz JAJ, Meszéna G: Adaptive Dynamics for Physiologically Structured Population Models. IIASA Interim Report IR-07-027 (2007).

No. 134 Ito H, Dieckmann U: A New Mechanism for Recurrent Adaptive Radiations. IIASA Interim Report IR-07-048 (2007). American Naturalist 170:E96-E111 (2007).

No. 135 Troost T, Kooi B, Dieckmann U: Joint evolution of predator body size and prey-size preference. IIASA Interim Report IR-07-050 (2007).

No. 136 Nowak MA, Sigmund K: How Populations Cohere: Five Rules for Cooperation. IIASA Interim Report IR-07052 (2007). May RM, McLean A (eds): Theoretical Ecology: Principles and Applications, Oxford UP, Oxford, pp. 716 (2007).

No. 137 Hauert C, Traulsen A, Brandt H, Nowak MA, Sigmund K: The Emergence of Altruistic Punishment: Via Freedom to Enforcement. IIASA Interim Report IR-07-053 (2007). Science 613:1905-1907 (2007)

No. 138 Sigmund K: Punish or Perish? Retaliation and Collaboration Among Humans. IIASA Interim Report IR-07-054 (2007). Trends in Ecology and Evolution 22:593-600 (2007).

No. 139 Kamo M, Sasaki A, Boots M: The Role of Trade-Off Shapes in the Evolution and Coexistence of Virulence in Spatial Host-Parasite Interactions: An Approximate Adaptive Dynamical Approach. IIASA Interim Report IR-07-061 (2007).

No. 140 Adams B, Sasaki A: The Influence of CrossImmunity on the Coexistence, Invasion and Evolution of Pathogen Strains. IIASA Interim Report IR-07-062 (2007).

No. 141 Metz JAJ, Mylius SD, Diekmann O: When Does Evolution Optimise? IIASA Interim Report IR-08-013 (2008). Evolutionary Ecology Research 10:629-654 (2008).

No. 142 Metz JAJ, Mylius SD, Diekmann O: Even in the Odd Cases When Evolution Optimises, Unrelated Population Dynamical Details May Shine Through in the ESS. IIASA Interim Report IR-08-014 (2008). Evolutionary Ecology Research 10:655-666 (2008).

No. 143 Pennings PS, Kopp M, Meszéna G, Dieckmann U, Hermisson J: An Analytically Tractable Model for Competitive Speciation. IIASA Interim Report IR-08-046 (2008). American Naturalist 171:E44-E71 (2008). 


\section{Contents}

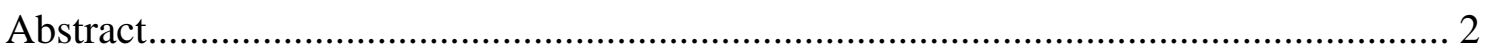

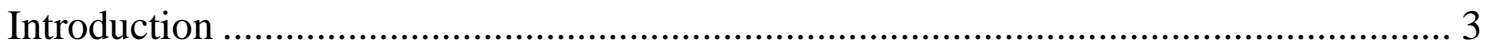

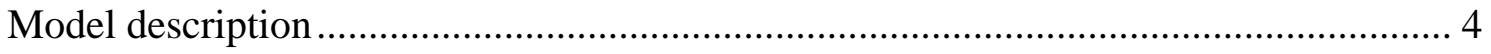

Stabilizing selection and competition...................................................................... 5

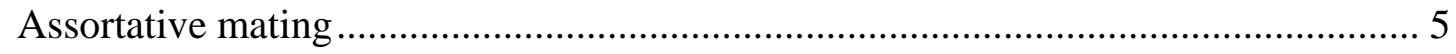

Population dynamics and fitness ........................................................................... 7

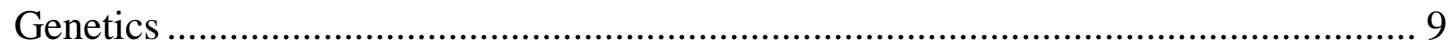

Analytical methods ............................................................................................. 10

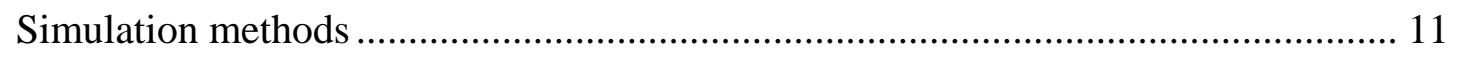

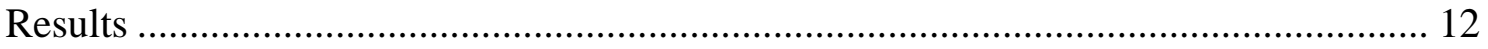

Evolution of female choosiness in the model without sexual selection ...................... 12

Evolution of female choosiness in the model with sexual selection ........................... 14

Stability of the ecological polymorphism................................................................ 18

Non-Gaussian competition functions ………………………………………….... 19

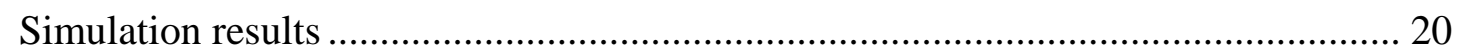

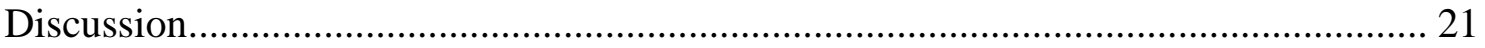

Natural and sexual selection ........................................................................... 24

Discussion of the modelling approach ................................................................... 25

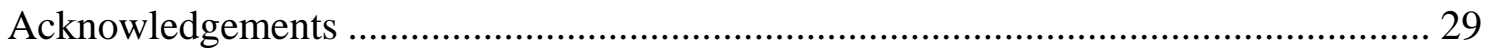

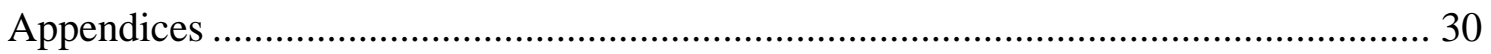

Appendix 1: Equations for the one-locus, two allele model........................................ 30

Appendix 2: Invasion analysis........................................................................... 32

Appendix 3: Evolutionary equilibria …………………....................................... 39

Appendix 4: General non-Gaussian competition functions........................................... 42

Appendix 5: Asymmetric model ........................................................................ 44

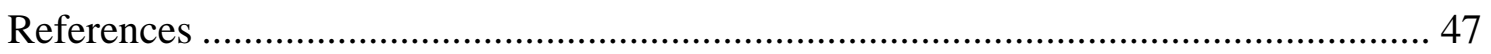




\title{
An analytically tractable model for competitive speciation
}

\author{
Pleuni S. Pennings*1, Michael Kopp*1, Géza Meszéna², \\ Ulf Dieckmann ${ }^{3}$, and Joachim Hermisson ${ }^{1}$
}

\footnotetext{
${ }^{1}$ Section of Evolutionary Biology, Department of Biology II, Ludwig-Maximilians University Munich, Großhaderner Str. 2, D-82152 Planegg-Martinsried, Germany.

${ }^{2}$ Department of Biological Physics, Eötvös University, Pázmany Péter sétány 1A, H-1117 Budapest, Hungary.

${ }^{3}$ Evolution and Ecology Program, International Institute for Applied Systems Analysis, Schlossplatz 1, A-2361 Laxenburg, Austria.

*These authors have contributed equally to this article.
}

Email addresses:

pennings@zi.biologie.uni-muenchen.de

kopp@zi.biologie.uni-muenchen.de

geza.meszena@elte.hu

dieckmann@iiasa.ac.at

hermisson@zi.biologie.uni-muenchen.de

Keywords. assortative mating, sympatric speciation, frequency-dependent selection, invasion fitness, sexual selection, population-genetic model 


\begin{abstract}
Several recent models have shown that frequency-dependent disruptive selection created by intraspecific competition can lead to the evolution of assortative mating and, thus, to competitive sympatric speciation. However, since most results from these models rely on limited numerical analyses, their generality has been subject to considerable debate. Here, we consider one of the standard models, the so-called Roughgarden model, with a simplified genetics where the selected trait is determined by a single diallelic locus. This model is sufficiently complex to maintain key properties of the general multilocus case, but still simple enough to allow for a comprehensive analytical treatment. By means of invasion fitness analysis, we describe the impact of all model parameters on the evolution of assortative mating. Depending on (1) the strength and (2) shape of stabilizing selection, (3) the strength and (4) shape of pairwise competition, (5) the shape of the mating function, and (6) the type of assortative mating, which may or may not lead to sexual selection, we find five different evolutionary regimes. In one of these regimes, the evolution of complete reproductive isolation is possible through arbitrarily small steps in the strength of assortative mating. Our approach provides a mechanistic understanding of several phenomena that have been found in previous models. The results demonstrate how, even in a simple model of competitive speciation, results depend in a complex way on ecological and genetic parameters.
\end{abstract}




\section{Introduction}

Interest in sympatric speciation has strongly increased in recent years. Empiricists have uncovered several likely examples of this mode of speciation in nature (Schliewen et al. 1994; Gíslason et al. 1999; Savolainen et al. 2006). At the same time, theoreticians have made substantial progress in understanding the potential mechanisms leading to sympatric lineage splitting (Dieckmann et al. 2004). One of these mechanisms is intraspecific competition. The idea of competitive speciation (Rosenzweig 1978) goes back to Darwin (Darwin 1859, pp. 113114) and has recently been studied in a series of models (e. g., Doebeli 1996; Dieckmann and Doebeli 1999; Matessi et al. 2001; Gavrilets 2004; Bürger et al. 2006; Bürger and Schneider 2006; Doebeli et al. 2007). In particular, Dieckmann and Doebeli (1999) used individualbased simulations of a competition model that goes back to MacArthur $(1969,1972)$ and Roughgarden (1972) to demonstrate that frequency-dependent disruptive selection on an ecological trait affecting resource competition can promote the evolution of assortative mating in a process similar to reinforcement. Sufficiently strong assortative mating then leads to reproductive isolation and speciation.

The fact that differential competition between phenotypes can induce frequency-dependent disruptive selection is commonly accepted (e. g., Seger 1985; Bolnick 2004a; Rueffler et al. 2006). What is controversial, however, is under exactly what circumstances such selection leads to the evolution of strong assortative mating. In particular, it has been questioned how much of the results from Dieckmann and Doebeli (1999) depend on model details, and this question has lead to intense debate (Doebeli and Dieckmann 2005; Doebeli et al. 2005; Gavrilets 2005; Polechová and Barton 2005; Waxman and Gavrilets 2005b). The reason for the continuing disagreement among evolutionary biologists lies in the non-intuitive nature of frequency-dependent selection and in the complexity of the models, which often allow only for a limited analysis based on computer simulations. For example, in the model by Dieckmann and Doebeli (1999), populations are subject to three different selective forces: stabilizing selection, frequency-dependent selection due to competition, and sexual selection due to assortative mating. In the genetically explicit version of the model, these forces act on two or three different traits, each of which is influenced by multiple loci.

Our aim in this study is to gain a more thorough understanding of the phenomena in competitive speciation models by a systematic analytical treatment. To this end, we discuss the 
evolution of assortative mating in a Roughgarden model with a simple genetic architecture, in which the ecological trait is determined by a single locus with two alleles. The same trait (or a pleiotropically related trait) serves as a marker for assortment. This corresponds to the first model of Dieckmann and Doebeli (1999), in which there is no separate marker trait for assortative mating. We thus concentrate on a "one-trait model" sensu Fry (2003), akin to the "one-allele model" in the original classification by Felsenstein (1981). Our approach extends the study by Matessi et al. (2001), who also investigated the evolution of assortative mating due to frequency-dependent disruptive selection acting on a single diallelic locus. In contrast to Matessi et al. (2001), we do not use a weak-selection approximation for the underlying fitness functions. This allows us to analyze a larger parameter space and selection pressures of any size. As it turns out, much more of the complexity of the original Dieckmann and Doebeli (1999) model is maintained in this way.

As our main analytical tool, we develop a simple invasion criterion that enables us to study the evolution of assortative mating in the entire parameter space. In addition, by comparing versions of the model with and without sexual selection, we clarify the roles of sexual and natural selection for competitive speciation. Our analysis reveals a remarkably complex structure: If the ecological locus remains polymorphic there are five qualitatively different evolutionary regimes, including two regimes previously described by Matessi et al. (2001). Our results show that predictions about the likelihood of competitive speciation require a detailed understanding of the underlying genetic and ecological factors - in models as well as in natural systems.

\section{Model description}

We consider a population of sexually reproducing hermaphrodites, which are characterized by two quantitative traits: an ecological trait that determines competition and, in the absence of competition, is under stabilizing selection, and a mating trait that determines the tendency for assortative mating with respect to the ecological trait. The timescale is chosen such that, per unit of time, each individual participates on average in one mating (playing either the male or the female role). 


\section{Stabilizing selection and competition}

Stabilizing selection in our model is the consequence of a carrying capacity $K$ that depends on the ecological phenotype $X$. We assume that $K(X)$ is maximal for $X=0$ but its shape can be quite general otherwise. In the original Roughgarden model (e. g., Roughgarden 1972; Dieckmann and Doebeli 1999), $K(X)$ has a Gaussian shape, that is,

$$
K(X)=K_{0} \exp \left(-\frac{X^{2}}{2 \sigma_{K}^{2}}\right)
$$

where $\sigma_{K}^{2}$ is the variance and the scaling parameter $K_{0}$ is the carrying capacity for individuals with phenotype $X=0$. An alternative choice for $K$ is a (truncated) quadratic function (Matessi et al. 2001).

Competition between a pair of individuals with phenotypes $X$ and $Y$ is described by a symmetric function $\gamma(X, Y)=\gamma(Y, X)$. Many models assume that $\gamma$ depends only on the phenotypic distance $|X-Y|$, as in the standard Gaussian competition function

$$
\gamma(X, Y)=\exp \left(-\frac{(X-Y)^{2}}{2 \sigma_{c}^{2}}\right)
$$

with variance $\sigma_{c}^{2}$, but we will also consider more general shapes. The total amount of competition experienced by an individual with phenotype $X$ is

$$
C(X)=\sum_{Y} \gamma(X, Y) N(Y)
$$

where $N(Y)$ is the number of individuals with phenotype $Y . C(X)$ can be seen as the "ecologically effective population size" experienced by the focal individual.

\section{Assortative mating}

Assortative mating is modeled as female choice (more precisely, choice by hermaphrodites in their female role) and is based on phenotypic similarity with respect to the ecological trait (Dieckmann and Doebeli 1999; Matessi et al. 2001; Bürger et al. 2006; Bürger and Schneider 2006). The probability $\mu(X, Y)$ that an encounter between a male and a choosy female leads to mating depends only on their phenotypic distance, $\mu(X, Y)=\mu(|X-Y|)$. We set $\mu(0)=1$ 
for normalization and assume $\mu(x) \leq 1$ (i.e., we do not allow for disassortative mating). The shape of the mating probability function depends on one or several variables that measure the degree of female choosiness. For most of our results, we will use a Gaussian mating function,

$$
\mu(|X-Y|)=\exp \left(-\frac{(X-Y)^{2}}{2 \sigma_{m}^{2}}\right)
$$

where the shape is determined by a single parameter for the variance $\sigma_{m}^{2}$. Here, small $\sigma_{m}^{2}$ corresponds to strong choosiness. The value(s) of the shape parameter(s) depend on the female mating genotype and can evolve.

Even though the aim of this study is to understand the evolution of female choosiness (i.e., the evolution of the shape of the mating probability function), most of the following analysis will be concerned with populations that are monomorphic with respect to the mating genotype. Polymorphic populations will only appear in the invasion analysis in Appendix 2, where the fate of rare mutants with a modified mating genotype is studied. To simplify our notation, we will therefore not include the explicit dependence on the mating genotype into the definition of the population-level variables. An appropriate notation to deal with different mating genotypes will be defined in Appendix 2, where it is needed.

We assume (for now) that the population is monomorphic for the mating genotype. Even in this case, assortative mating may lead to genotype-specific mating rates $\phi=\left(\phi_{\text {female }}+\phi_{\text {male }}\right) / 2$ on the population level, where female and male mating rates are separately defined as

$$
\begin{aligned}
\phi_{\text {female }}(X) & =\sum_{Y} N(Y) \mu(X, Y) M(X), \\
\phi_{\text {male }}(X) & =\sum_{Y} N(Y) \mu(X, Y) M(Y) .
\end{aligned}
$$

(Note that Matessi et al. (2001) use the term mating rate in a different sense.) Here, we have introduced additional factors $M(X)$, which describe female mating activity. The idea is that females with different genotypes may have different encounter rates with males. In general, $M$ may also depend on the genotype distribution in the population. By different choices of $M$, we can model different types of assortative mating in a common framework. 
In particular, we will consider two models in the following. Both models assume that choosiness has no direct costs for females. This means that the mating rate of an individual female does not depend on her mating genotype, because differences in the mating probabilities $\mu$ are compensated by differences in the mating activities $M$. Even with this assumption, assortative mating may or may not lead to sexual selection, depending on whether or not the mating rates $\phi$ differ between the ecological genotypes:

Model 1: No sexual selection. In our first model, assortative mating does not lead to sexual selection on the ecological trait. The mating activity factors $M(X)$ are chosen such that all phenotypes contribute to the offspring pool according to their frequency in the mating population. This is achieved by setting $\phi(X)=1$ for all $X$ and solving the resultant linear equation system (see Appendix 1).

Model 2: Sexual selection against rare males. Our second model follows Dieckmann and Doebeli (1999). Here, $M(X)$ is thus chosen such that all females have the same normalized mating rate $\phi_{\text {female }}(X)=1$ (see Appendix 1). This means that each female is guaranteed to eventually find a mate whom she does not reject. In contrast, male mating rates are not normalized but depend on $X$. Therefore, female choice entails sexual selection on males and leads to a disadvantage for rare ecological phenotypes. Note that, in this paper, we only study sexual selection that arises as a consequence of assortative mating based on the ecological trait (see Kirkpatrick and Nuismer 2004; Gourbière 2004). We do not consider sexual selection due to female preference for male display traits, which by itself has been discussed as a potential cause of sympatric speciation via Fisherian run-away processes (Higashi et al. 1999; van Doorn et al. 2004; Arnegard and Kondrashov 2004).

\section{Population dynamics and fitness}

In the following, we assume that time is continuous (generations are overlapping) and population sizes are large enough to ignore stochastic processes such as genetic drift. All mated individuals are assumed to produce an equal number of offspring, $r$. Therefore, the fertility of individuals with phenotype $X$ is $r \phi(X)$, that is, all differences in fertility are due to differences in the mating rates. While fertility is the rate at which a phenotype gives birth, the 
birth rate $B(X)$ is the rate at which individuals with phenotype $X$ are born. For our model,

$$
B(X)=r \sum_{Y, Z} N(Y) N(Z) \mu(Y, Z) M(Z) R_{Y Z \rightarrow X},
$$

where $R_{Y Z \rightarrow X}=R_{Z Y \rightarrow X}$ is the probability (depending on genetic details) that a mating between $Y$ and $Z$ individuals produces $X$ offspring.

We can now write down the population dynamics, which follow a Lotka-Volterra model,

$$
\dot{N}(X)=B(X)-N(X) d(X)
$$

where the per-capita death rate $d(X)$ is equal to the ratio of the effective population size and the carrying capacity,

$$
d(X)=\frac{C(X)}{K(X)}
$$

This model is commonly interpreted in terms of competition among phenotypically variable consumers for a continuum of (demographically rapid) resources (e. g., MacArthur 1969; Ackermann and Doebeli 2004). The canonical example is birds with different beak sizes specializing on differently sized seeds (Schoener 1965). Then, $K(X)$ describes the availability of resources favored by consumers with phenotype $X$, and $\gamma(X, Y)$ describes the overlap in resource use between two individuals with phenotypes $X$ and $Y$. The standard deviation $\sigma_{c}$ determines the range of resources used by a single individual, that is, the individual niche width (Bolnick et al. 2003). If $\sigma_{c}$ is small, selection arising from competition is strongly frequency-dependent.

Finally, the (Malthusian) fitness of individuals with phenotype $X$ is given by the rate at which individuals give birth minus the rate at which they die, that is, by

$$
W(X)=r \phi(X)-d(X)
$$

In other words, fitness can be separated into two components, one related to mating success and one to survival. In the following, we will use the notion that $d(X)$ describes natural selection due to stabilizing selection and resource competition, whereas $\phi(X)$ describes sexual selection due to female choosiness. Equation (9) will be the basis for the invasion analysis 
that allows us to study the evolution of female choosiness. In the remainder of this paper, we will set $r=1$. This is without loss of generality, because a different value of $r$ can be accounted for by changing the timescale in equation (7) and rescaling the carrying capacity parameter $K_{0}$ to $r K_{0}$.

\section{Genetics}

To allow for a detailed analytical treatment, we now make the simplifying assumption that the ecological trait is determined by a single diploid locus with two alleles, '+' and '- '. We ignore environmental variation and assume that the allelic effects are symmetric with respect to the maximum of the carrying capacity $K(X)$. Individuals with genotype $(+/+)$ have phenotype $x$, individuals with genotype $(+/-)$ have phenotype 0 , and individuals with genotype $(-/-)$ have phenotype $-x$. We call $x$ the allelic effect of the ecological locus.

As there are only three ecological phenotypes, we can use a simplified notation. We will denote the numbers of individuals carrying these genotypes by $N_{\text {hom }}^{+}, N_{\text {het }}$, and $N_{\text {hom }}^{-}$, respectively, where 'hom' and 'het' stand for homozygotes and heterozygotes. Similarly, we will use $K_{\text {hom }}^{ \pm}=K( \pm x)$ and $K_{\text {het }}=K(0)=K_{0}$, and analogous subscripts for the other parameters. Furthermore, we will denote average parameter values over both homozygote classes by, for example, $N_{\text {hom }}=\left(N_{\text {hom }}^{+}+N_{\text {hom }}^{-}\right) / 2$ and $K_{\text {hom }}=\left(K_{\text {hom }}^{+}+K_{\text {hom }}^{-}\right) / 2$. The strength of stabilizing selection will be described by two (dimensionless) variables

$$
k^{ \pm}=1-\frac{K_{\mathrm{hom}}^{ \pm}}{K_{\mathrm{het}}}
$$

and the competition and mating functions by three and two values, respectively,

$$
\begin{aligned}
c^{ \pm} & =1-\gamma( \pm x, 0), \\
c^{\prime} & =1-\gamma(-x, x), \\
m & =1-\mu(x), \\
m^{\prime} & =1-\mu(2 x) .
\end{aligned}
$$

Frequency dependence induced by competition increases with $c^{ \pm}$and $c^{\prime}$, which parametrize the niche shape. Female choosiness increases with $m$ and $m^{\prime}$, which measure the probabilities for females to reject males with a different phenotype. This parametrization makes it possible 
to consider very general shapes of the functions $K, \gamma$, and $\mu$. All we assume in our analytical derivations is that $k^{ \pm}, c^{ \pm}, c^{\prime}, m, m^{\prime} \in[0,1]$ and that $m^{\prime}$ is a non-decreasing function of $m$ that extends from $m^{\prime}=0$ for $m=0$ to $m^{\prime}=1$ for $m=1$. This implies that mating modifiers cannot change $m$ and $m^{\prime}$ in opposite directions. (For all figures, a Gaussian mating function with $\left(1-m^{\prime}\right)=(1-m)^{4}$ was assumed.) Explicit expressions for the mating rates and fitness components in terms of these parameters, as well as a table summarizing the major model parameters, are given in Appendix 1.

For simplicity, we will restrict our presentation in the body of this paper to symmetric cases with $k^{+}=k^{-}=k$ and $c^{+}=c^{-}=c$. Furthermore, if not otherwise stated, we will employ the Gaussian functions (2) and (4) for $\gamma$ and $\mu$, where $c^{\prime}=1-(1-c)^{4}$ and $m^{\prime}=1-(1-m)^{4}$. The general asymmetric case is treated in Appendices 2 and 5. A major simplification arising from the symmetry assumption is that, in a population with a monomorphic mating genotype, the polymorphic equilibrium of equation (7) is also symmetric with $N_{\text {hom }}^{+}=N_{\text {hom }}^{-}=N_{\text {hom }}$. At this equilibrium, the genotype distribution is fully described by the ratio of heterozygotes to homozygotes,

$$
n=\frac{N_{\text {het }}}{N_{\text {hom }}}
$$

which equals 0 for complete reproductive isolation and 2 at Hardy-Weinberg equilibrium.

Parameters of assortative mating are determined by the alleles at (one or several) modifier loci. Since we assume that there is no cost of choosiness, modifier alleles do not change the fitness of their carriers. They are therefore pure modifiers in the sense of classical modifier theory (Karlin and McGregor 1974). In our analytical treatment, we assume that modifier mutations are rare and that at most a single mating modifier (at recombination distance $\rho$ from the ecological locus) segregates at a given time. We also assume that individual modifiers have a small effect, but we do not need to specify the genetic basis of the mating trait any further at this point.

\section{Analytical methods}

The main goal of this study is to understand the evolution of female choosiness. To this end, we use an invasion fitness approach for mating modifiers (Karlin and McGregor 1974; Metz 
et al. 1992) and ask when a rare mutant with higher or lower choosiness can invade a resident population with a monomorphic mating genotype. Our main tool is the following

Invasion criterion. Assume that the population is at a (symmetric or asymmetric) polymorphic equilibrium of the ecological locus. A mutant modifier allele for stronger female choosiness (larger $m$ and $m^{\prime}$ ) is able to invade a resident population with monomorphic mating genotype if and only if the homozygote residents have higher fitness than the heterozygote residents, $W_{\text {hom }}>W_{\text {het }}$.Vice versa, a mutant with smaller $m$ and $m^{\prime}$ is able to invade if and only if $W_{\text {hom }}<W_{\text {het }}$.

Thus, the direction of selection on female choosiness is determined by the sign of $\Delta_{W}=$ $W_{\text {hom }}-W_{\text {het }}$, and an evolutionary equilibrium is reached if $\Delta_{W}=0$. This criterion is independent of potential linkage between mating modifiers and the ecological locus. A formal proof is given in Appendix 2. Intuitively, the idea is as follows: Since we have assumed that there is no cost of choosiness, the mating strategy of the mutant has no direct influence on its fitness. However, the mating genotype of females determines the distribution of mutant genotypes in the next generation (and beyond). Since females with a higher choosiness than the residents will have proportionally more homozygous offspring (with respect to the ecological genotype), corresponding mutants are favored for $\Delta_{W}>0$, and vice versa. In accordance with equation (9), $\Delta_{W}$ can be written as the sum of two components stemming from sexual and natural selection, respectively,

$$
\Delta_{W}=\Delta_{\phi}+\Delta_{d}
$$

where $\Delta_{\phi}=\phi_{\text {hom }}-\phi_{\text {het }}$ and $\Delta_{d}=-\left(d_{\text {hom }}-d_{\text {het }}\right)$.

\section{Simulation methods}

In addition to the invasion analysis, we carried out individual-based simulations. The aim of the simulations was twofold: First, to check the validity of the analytical results for finite populations (i. e., in the presence of genetic drift and demographic stochasticity), and second, to see how the likelihood of speciation depends on the genetic architecture of the mating trait. For this purpose, we now specify that female choosiness is determined by one diploid, additive 
locus with a continuum of possible alleles. New alleles are created by mutations occurring at rate $u$ per individual and generation and follow a stepwise mutation model at the scale of $m$ (e. g., if the step size is 0.1 , an allele coding for $m=0.5$ can mutate to $m=0.4$ or $m=0.6$, which of course leads to a non-constant step size at the scale of $\sigma_{m}$ ). There are no mutations at the ecological locus. The ecological locus and the choosiness locus are unlinked. Initial populations are polymorphic at the ecological locus (with allele frequencies 0.5) but monomorphic at the choosiness locus. Gaussian functions for $K, \gamma$, and $\mu$ are assumed throughout. Simulations are run for a maximum of 100, 000 generations, where one generation is defined by $2 N$ birth or death events (where $N$ stands for total population size). Speciation, in the simulations, is defined as the absence of heterozygotes.

\section{Results}

In the following, we investigate the evolution of female choosiness, starting with the symmetric model without sexual selection. We find three evolutionary regimes, which are then complemented by two further regimes identified in the symmetric model with sexual selection. Next, we examine the stability of ecological polymorphisms and the implications of non-Gaussian competition functions, before presenting simulation results to corroborate and complement our analytical findings. Results for asymmetric models are presented in Appendix 5.

\section{Evolution of female choosiness in the model without sexual selection}

In the model without sexual selection (i. e., with $\phi=1$ ), the evolution of female choosiness is determined by natural selection alone. As shown in Appendix 3.1, for each parameter combination, natural selection favors a unique value $\hat{n}$ of the heterozygote-to-homozygote ratio $n$ (eq. A36). Female choosiness then evolves in such a way that $n$ matches $\hat{n}$, within the constraints that $m$ and $m^{\prime}$ must be between 0 and 1 (because we do not allow for disassortative mating). This leads to three qualitatively different evolutionary regimes (Fig. 1a, b): 
Random-mating ( $\mathbf{R})$ regime. The population evolves to random mating $\left(m=m^{\prime}=0\right)$ if $\hat{n} \geq 2$, that is, if

$$
k \geq \frac{c^{\prime}}{4-2 c}
$$

(solid line in Fig. 1a, b). This is the case if stabilizing selection is strong $(k, x$ large) or competition induces weak frequency dependence ( $c$ small, $\sigma_{c}$ large). Random mating is stable for any value of $c$ if $k>0.5$, that is, if the carrying capacity of homozygotes is less than half of that of heterozygotes.

Complete-isolation (C) regime. The population evolves to complete reproductive isolation $\left(m=m^{\prime}=1\right)$ if $\hat{n} \leq 0$, that is if

$$
k \leq \frac{c^{\prime}-2 c}{2-2 c}
$$

(dashed line in Figure 1a, b). This is the case if stabilizing selection is weak $(k, x$ small) and competition induces moderate frequency dependence (intermediate $c$ and $\sigma_{c}$ ).

Partial-isolation (P) regime. If neither condition (13) nor condition (14) is fulfilled, the population evolves to an intermediate level of choosiness, entailing partial reproductive isolation.

Two observations from Figure 1 are noteworthy. First, the often-cited condition for the emergence of frequency-dependent disruptive selection in the Gaussian model, $\sigma_{c}<\sigma_{K}$ (or, equivalently, $c>k$; Christiansen and Loeschcke 1980; Dieckmann and Doebeli 1999), is necessary but not sufficient for complete (or even partial) isolation in this model. The reason is the rigid genetic architecture that results from the allelic effect $x$ being constant: Even if disruptive selection favors lineage splitting in a continuous genotype space, this will not happen if $x$ is too large and, consequently, stabilizing selection against homozygotes is too strong. The above condition is reproduced in the limit $x \rightarrow 0$, as can be seen from equations (13) and (14) with $c^{\prime}=1-(1-c)^{4} \rightarrow 4 c$. Second, partial isolation replaces complete isolation if competition induces strong frequency dependence (large $c$ ). This is because, if competition among homozygotes and heterozygotes becomes very weak, heterozygotes will occupy a third ecological niche. 

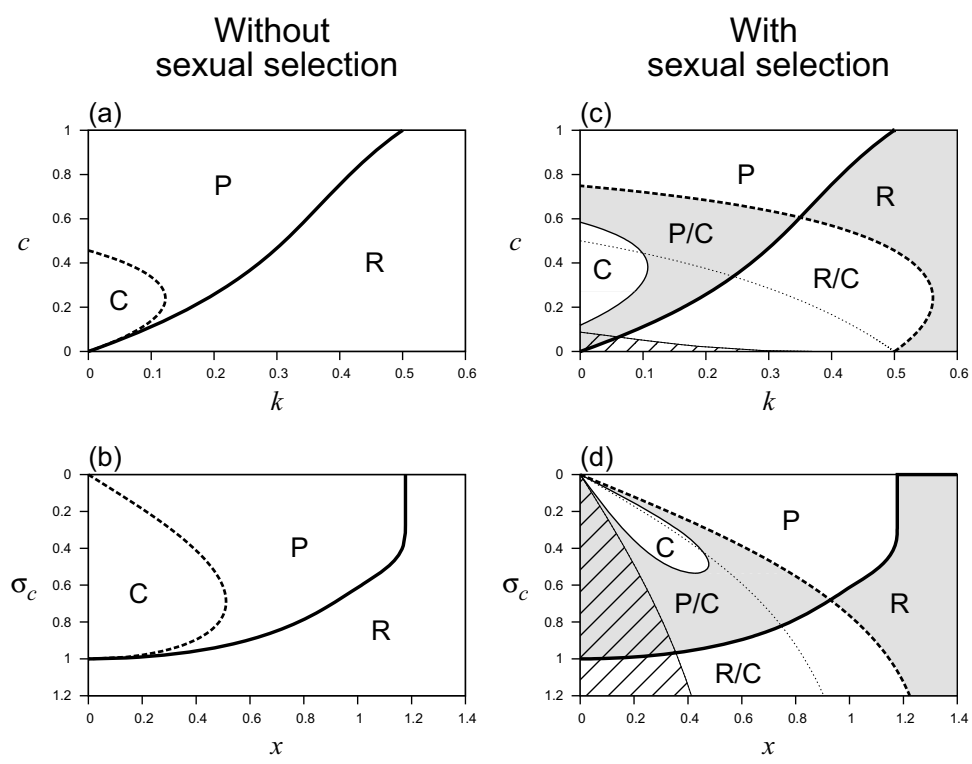

Figure 1: Evolutionary regimes for female choosiness in the Gaussian models without and with sexual selection. (a) and (c) show the results as a function of $k$ and $c$ (which are direct measures of selection strength), whereas (b) and (d) show the same results in the alternative parameter space spanned by the ecological parameter $\sigma_{c}$ and the genetic parameter $x$. Note that the orientation of the $\sigma_{c}$ axis is downwards. All plots assume $\sigma_{K}=1$ (without loss of generality) and Gaussian fitness and mating functions (eq. 1, 2, and 4). In the model without sexual selection, (a) and (b), there are three regimes: complete-isolation $(\mathrm{C})$, partial-isolation $(\mathrm{P})$, and random-mating $(\mathrm{R})$. The boundaries of the $\mathrm{R}$ regime (solid line) and the $\mathrm{C}$ regime (dashed line) are defined by (13) and (14). With sexual selection, (c) and (d), there are two additional bistable regimes: the $\mathrm{R} / \mathrm{C}$ regime, where, depending on initial conditions, the population evolves either random mating or complete isolation, and the $\mathrm{P} / \mathrm{C}$ regime, where it evolves either partial or complete isolation. Local stability of random mating (to the right of the solid line) is identical to the case without sexual selection. Stability of complete isolation (to the left of the thick dashed line) is now given by (15). In the hatched area, the polymorphic equilibrium at the ecological locus is unstable for intermediate values of choosiness. To the left of the thin dotted line, the monomorphic equilibria are locally stable if choosiness is sufficiently large (see 18).

\section{Evolution of female choosiness in the model with sexual selection}

In the model with sexual selection, homozygote and heterozygote males may differ in their mating rates. Therefore, evolution of choosiness is determined by both natural and sexual selection. We analyze the evolutionary equilibria using the invasion criterion based on the sign of $\Delta_{W}=W_{\text {hom }}-W_{\text {het }}$ (Figures 2); all derivations for this section are given in Appendix 3.2. As in the model without sexual selection, evolutionary equilibria can be characterized by random mating, partial isolation, or complete isolation. Random mating is evolutionarily stable under the same condition as in the model without sexual selection, given by inequality (13), because sexual selection does not act if mating is random. Complete isolation $\left(m=m^{\prime}=1\right)$ is stable 


$$
k<\frac{2-4 c+c^{\prime}-\left(2-c^{\prime}\right)(\sqrt{1+2 / \kappa}-1) \kappa}{4-4 c}
$$

where $\kappa=\lim _{m, m^{\prime} \rightarrow 1}\left(1-m^{\prime}\right) /(1-m)$. For a Gaussian mating function with $\left(1-m^{\prime}\right)=$ $(1-m)^{4}, \kappa=0$, and condition (15) is considerably less strict than condition (14) in the model without sexual selection (compare thick dashed lines in Figure 1c and d versus a and b). In general, inequality (15) shows that the evolutionary equilibria depend on the shape of the mating function. Finally, the invasion analysis reveals that, in addition to the randommating $(\mathrm{R})$, partial- isolation $(\mathrm{P})$, and complete-isolation $(\mathrm{C})$ regimes, where there is only one possible evolutionary outcome, the model with sexual selection has two additional bistable regimes (Fig. 1c, d, 2, 3):

Bistable regime with random mating or complete isolation $(\mathrm{R} / \mathrm{C})$. In a large part of parameter space (small $c$ and intermediate $k$ ), random mating and complete isolation are both locally stable. The evolutionary outcome then depends on the initial level of choosiness.

Bistable regime with partial isolation or complete isolation $(\mathrm{P} / \mathrm{C})$. In part of the parameter region where random mating is unstable and complete isolation is stable, there are two additional intermediate equilibria, one stable and one unstable. A population that starts at random mating and whose choosiness evolves in small steps will reach the stable intermediate equilibrium, and only a population that starts with an already high level of choosiness (or evolves in large steps) can evolve to complete isolation. This threshold phenomenon has previously been described by Doebeli (1996) and Matessi et al. (2001).

\section{Natural versus sexual selection}

To understand the evolution of female choosiness in this model, we need to consider the joint action of natural and sexual selection as the population moves from random mating to complete isolation. Natural selection for homozygotes (favoring assortative mating) is described by $\Delta_{d}$ (eq. 12 and A8), which satisfies

$$
\Delta_{d} \propto(c-k) n+c^{\prime}-2 c-2 k(1-c) .
$$


Random mating

(R)

$\Delta$

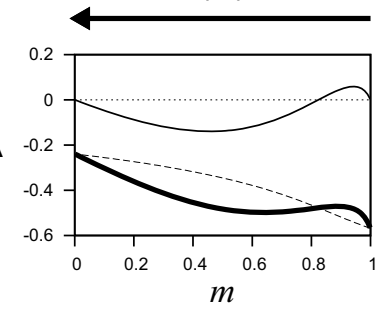

Random mating or complete isolation $(\mathrm{R} / \mathrm{C})$

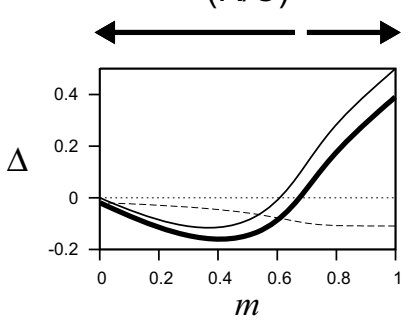

Partial isolation $(\mathrm{P})$

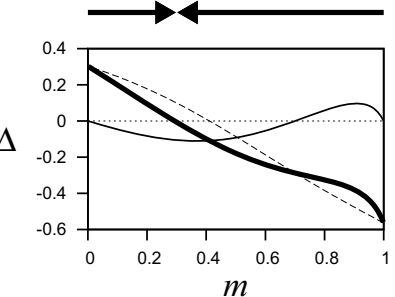

Partial isolation or complete isolation $(\mathrm{P} / \mathrm{C})$

$\Delta$

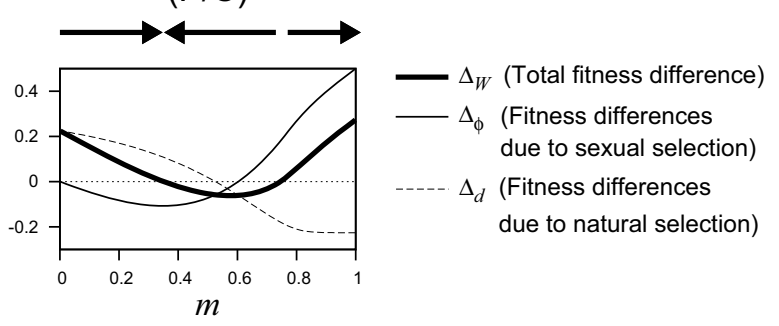

Complete isolation

(C)

$\Delta$

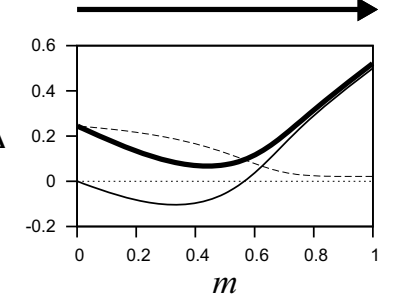

Figure 2: Selection on female choosiness in the five evolutionary regimes of the model with sexual selection. The plots show the fitness advantage of homozygotes, $\Delta_{W}=W_{\text {hom }}-W_{\text {het }}$ (thick solid line), as a function of female choosiness $m . \Delta_{W}$ is the sum of two components, $\Delta_{\phi}=\phi_{\mathrm{hom}}-\phi_{\mathrm{het}}$ (thin solid line) and $\Delta_{d}=-\left(d_{\mathrm{hom}}-\right.$ $d_{\text {het }}$ ) (dashed line), which describe the direction of sexual selection and natural selection, respectively. Arrows indicate the resultant direction of selection on $m$. Parameters: $x=1.2, \sigma_{c}=0.9(\mathrm{R}) ; x=0.4, \sigma_{c}=0.2(\mathrm{P})$; $x=0.3, \sigma_{c}=0.3(\mathrm{C}) ; x=0.7, \sigma_{c}=0.9(\mathrm{R} / \mathrm{C}) ; x=0.5, \sigma_{c}=0.4(\mathrm{P} / \mathrm{C})$. Fitness and mating functions are assumed to be Gaussian.

For $c>k$, selection on the ecological phenotype is disruptive and $\Delta_{d}$ is monotonically increasing with $n$ (i. e., selection for homozygotes increases with the frequency of heterozygotes). This means that competition leads to negative frequency dependence, so that rare phenotypes are favored. Since $n$ generally decreases with increasing $m$ and $m^{\prime}$, this explains the shape of $\Delta_{d}$ in Figure 2 (where $c>k$ is always fulfilled). In contrast, for $c<k$, natural selection on the ecological phenotypes has a net stabilizing effect, and $\Delta_{d}$ is decreasing with $n$. Intuitively, this means that an increase in the number of heterozygotes has a stronger negative effect on the homozygotes (which have a lower carrying capacity) than on the other heterozygotes. In other words, natural selection in this case induces positive frequency dependence.

Sexual selection on the ecological phenotype is always positively frequency-dependent (frequent phenotypes are more likely to find a mate). It favors homozygotes when they are common and disfavors them when they are rare. For given $n, \Delta_{\phi}$ (see eq. 12) is positive (favoring homozygotes) if and only if 


$$
n<2-\frac{m^{\prime}}{m}
$$

Under the reasonable assumption that $m^{\prime} \geq m$, assortative mating must be strong enough for the phenotype distribution to become bimodal $(n<1)$ before sexual selection favors increased choosiness. Similarly, increased choosiness is never favored as long as the mating function is concave $\left(m^{\prime}>2 m\right.$, which is possible only for $\left.m<0.5\right)$.

The thin solid line in Figure 2 shows the fitness difference caused by sexual selection, $\Delta_{\phi}$, as a function of $m$ (and at the equilibrium value of $n$ ). $\Delta_{\phi}$ equals zero at random mating and initially decreases (i. e., becomes negative) with increasing $m$, thus acting against further increase in choosiness. This is because, at low $m$, heterozygotes are sufficiently common to be favored by sexual selection. Near complete isolation, however, where $n$ is small and $m$ and $m^{\prime}$ are close to $1, \Delta_{\phi}$ is always positive. For parameter values where complete isolation is evolutionarily stable (and assuming a Gaussian mating function), it reaches its maximal possible value of $1 / 2$ at $m=m^{\prime}=1$. For parameter values where complete isolation is unstable, $\Delta_{\phi}$ at $m=1$ drops back to zero (see Appendix 3.2 for more details).

Thus, sexual selection can have two different effects (see Fig. 2): (1) For low $m$ and $m^{\prime}$, sexual selection creates a barrier against further increase of choosiness. For complete reproductive isolation to evolve, natural selection from competition must overcome this barrier. Otherwise, the population gets stuck at an intermediate equilibrium (as in the $\mathrm{P} / \mathrm{C}$ regime), where natural selection (favoring increased choosiness) balances with sexual selection (favoring decreased choosiness). (2) For already high levels of choosiness (leading to a bimodal phenotype distribution), sexual selection is a potent force that can drive populations towards complete reproductive isolation. Since $\Delta_{\phi}$ reaches a maximum at $m=m^{\prime}=1$ for $n \rightarrow 0$, whereas $\Delta_{d}$ decreases (assuming $c>k$ ), the relative importance of sexual versus natural selection increases towards this limit. This is particularly evident in the $\mathrm{R} / \mathrm{C}$ regime, where sexual selection can maintain complete isolation even though natural selection favors random mating. 


\section{Stability of the ecological polymorphism}

So far, we have assumed that the ecological locus is always at a polymorphic symmetric equilibrium, with the proportion of heterozygotes determined by female choosiness. This equilibrium is, indeed, always favored by natural selection (for $c>k$ by frequency-dependent disruptive selection, and for $c<k$ due to heterozygote advantage). However, monomorphic equilibria (containing either only the + or the - allele) may become stable due to the positive frequency dependence of sexual selection. Obviously, evolution of complete isolation is only possible if the ecological locus remains polymorphic. In Appendix 3.2, we show that the monomorphic equilibria are locally stable if

$$
\frac{m}{2}>c+k-c k
$$

that is, if sexual selection from female choosiness is strong enough relative to natural selection (thin dotted line in Figure 1c and d).

Whether the polymorphic equilibrium is locally stable can be determined numerically by standard linear stability analysis (i. e., by numerically calculating the eigenvalues of system A10). When the polymorphic and the monomorphic equilibria are both locally stable, their respective domains of attractions can be estimated by iterating system (A10) with different initial allele frequencies. The polymorphic equilibrium is unstable for small $x$ and intermediate $m$ (dark grey area in Fig. 3; Bürger and Schneider 2006; Bürger et al. 2006). This parameter range does not overlap with the domain of the complete isolation regime (see the hatched area in Fig. 1c, d). A polymorphic population evolving in the complete isolation regime may enter an area where the monomorphic equilibria are locally stable. However, their domain of attraction is very small (very light grey area to the left of the dashed line in Fig. 3). Therefore, a sufficiently large population will almost certainly remain polymorphic and safely reach complete isolation. For the $\mathrm{P} / \mathrm{C}$ regime, our numerical analysis for the Gaussian model shows that a polymorphic population starting from random mating and evolving in small steps will always reach the stable intermediate value of $m$ before the monomorphic equilibria become locally stable (see Fig. 3, where the thick black line lies below the grey area). Matessi et al. (2001) found similar results for a quadratic model. 

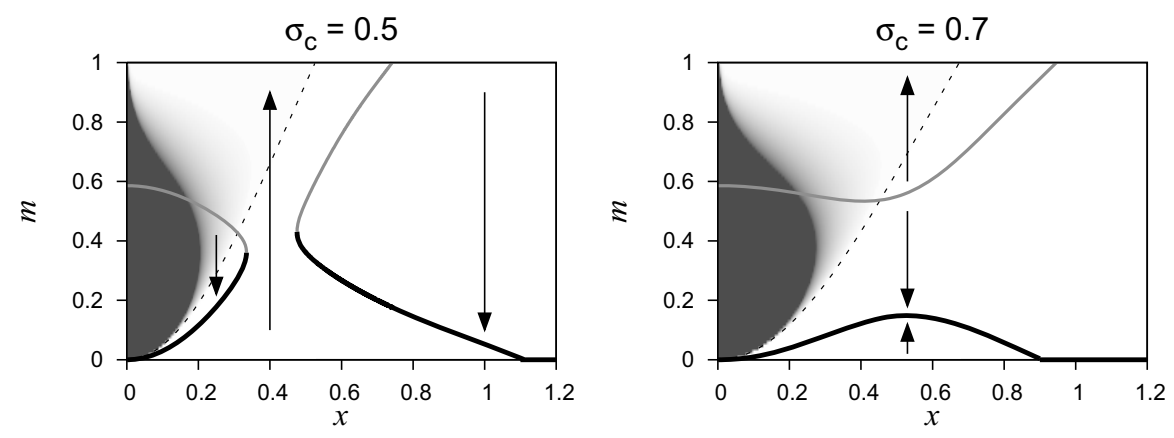

Figure 3: Stability of the ecological polymorphism and evolutionary equilibria of female choosiness $m$ as a function of the allelic effect $x$ (assuming Gaussian fitness and mating functions). In the dark grey area, the polymorphic equilibrium is unstable. To the left of the dashed line, the monomorphic equilibrium is locally stable (see inequality 18). Shades of grey indicate the size of the domain of attraction of the polymorphic equilibrium in terms of the frequency of the + allele (white: polymorphic equilibrium is globally stable; dark grey: polymorphic equilibrium is unstable). Black lines show stable equilibria and gray lines unstable equilibria for $m$. Arrows indicate the direction of selection on $m$. Each plot illustrates the succession of evolutionary regimes along a horizontal line in Figure 1d. For example, the complete isolation regime exists for intermediate $x$ if $\sigma_{c}=0.5$ but not if $\sigma_{c}=0.7$. Note that evolution of $m$ will come to a halt if the population moves into the dark grey area where the polymorphism at the ecological locus is unstable.

\section{Non-Gaussian competition functions}

Figures 1 to 3 are based on the Gaussian functions (1), (2), and (4), but the analytical results in equations (13) to (18) hold for arbitrary symmetric fitness functions. In the following, we analyse, in more detail, the influence of the shape of the competition function. In the symmetric model, this shape is characterized by the two parameters $c$ and $c^{\prime}$. In the Gaussian model (eq. 2), $1-c^{\prime}=(1-c)^{4}$, but other relationships are no less possible. Assuming that the strength of competition does not increase with phenotypic distance $\left(c^{\prime} \geq c\right)$, two extreme cases are given by $c^{\prime}=c$ and $c^{\prime}=1$. In the first case, different homozygotes compete as strongly with each other as they do with the heterozygotes. Therefore, competition induces less strong frequency dependence than in the Gaussian model. In the second case, different homozygotes do not compete at all, and competition induces stronger frequency dependence than in the Gaussian model.

As one should expect, weaker frequency dependence $\left(c^{\prime}=c\right)$ makes reaching complete isolation less likely. Indeed, in this case, the complete-isolation regime is entirely missing (Fig. 4a and c). In contrast, if frequency-dependent selection is stronger than in the Gaussian model 

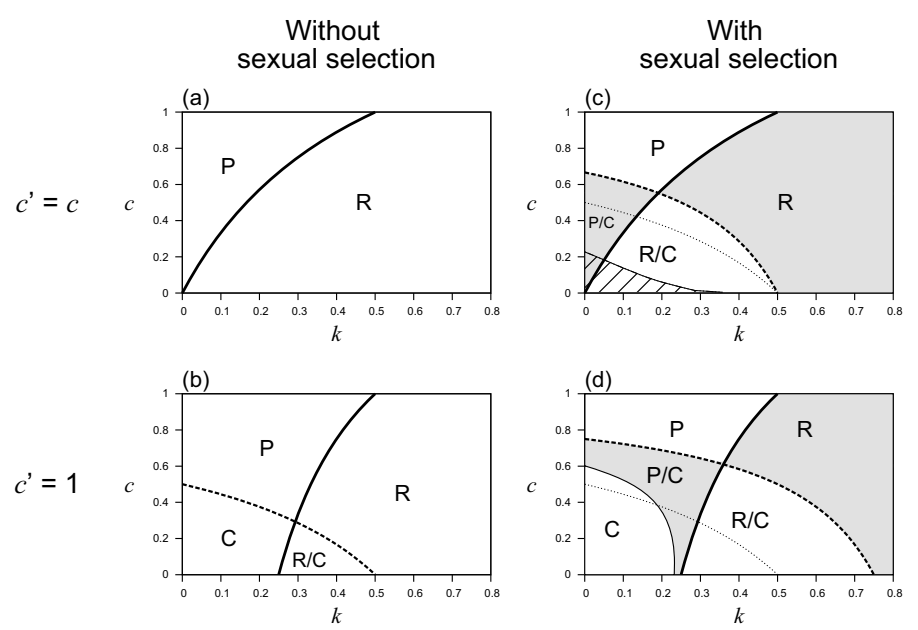

Figure 4: Evolutionary regimes for female choosiness with non-Gaussian competition functions. In (a) and (c) competition between different homozygotes is as strong as competition between homozygotes and heterozygotes $\left(c^{\prime}=c\right)$. Without sexual selection, complete isolation cannot be evolutionarily stable. With sexual selection, complete isolation can be an evolutionarily stable state, but it cannot evolve from random mating in small steps (i. e., the $\mathrm{C}$ regime is absent, and only the bistable $\mathrm{R} / \mathrm{C}$ and $\mathrm{P} / \mathrm{C}$ regimes exist). In (b) and $(\mathbf{d})$, homozygotes with different phenotypes do not compete with each other $\left(c^{\prime}=1\right)$. The parameter range corresponding to the complete-isolation regime is larger than in the Gaussian model. For more details, see Figure 1.

$\left(c^{\prime}=1\right)$, the domain of the complete-isolation regime expands (Figure $4 \mathrm{~b}$ and $\mathrm{d}$ ) and now includes areas with arbitrarily small $c$. Furthermore, a bistable regime $(\mathrm{R} / \mathrm{C})$ exists also in the absence of sexual selection (but only for $c<k$ ). The bistability can be explained by the fact that, for $c<k$, natural selection induces positive frequency dependence (see above). Finally, the polymorphic equilibrium of the ecological locus is always locally stable. A more general analysis for arbitrary values of $c$ and $c^{\prime}$ is given in Appendix 4. The influence of asymmetry in the carrying capacity and the competition function is discussed in Appendix 5.

\section{Simulation results}

Our analytical results for the model with sexual selection are qualitatively confirmed by the individual-based simulations. In particular, we did not find any new equilibria or evolutionary regimes. In the random-mating, partial-isolation, and complete-isolation regimes, the expected equilibrium was always reached, independent of initial conditions. For the complete-isolation regime, simulations with different parameter combinations showed that, for given mutational step size, the time required for reaching complete isolation depends only 
on the product of population size and mutation rate (results not shown), as predicted by the canonical equation of adaptive dynamics (Dieckmann and Law 1996). In the bistable R/C regime, the outcome of the simulations depends on initial conditions, as predicted by our analytical results.

In the $\mathrm{P} / \mathrm{C}$ regime, a finite population that starts at random mating can evolve towards three possible equilibria. (1) It can lose the polymorphism at the ecological locus, (2) it can end up at the stable equilibrium with intermediate $m$, and (3) it can end up at the stable equilibrium corresponding to complete isolation $(m=1)$, thereby jumping over the unstable intermediate equilibrium (see Figure 2). For a very small population $\left(K_{0}=500\right)$, the polymorphism at the ecological locus is always lost for parameter combinations for which the polymorphic equilibrium becomes unstable for intermediate $m$ (Figure 5 , see also the hatched area in Figure 1c, d). Outside of these areas, the population often evolves to an intermediate degree of choosiness. Evolution of complete isolation is most likely for parameter combinations close to the complete- isolation regime. At these parameters, the valley of $m$ values within which selection acts against homozygotes (i. e., against an increase in choosiness; $\left.\Delta_{W}<0\right)$ is narrow, such that the jump needed to reach complete isolation is relatively easy. The probability of reaching complete isolation in this manner also strongly depends on the mutational parameters. In our model, reaching complete isolation requires high mutation rates and relatively large mutational effects (Figure 6). Furthermore, for given mutational parameters, the probability of jumping does not increase with increasing population size (results not shown).

\section{Discussion}

Under what conditions can intraspecific competition lead to sympatric speciation? - This question has been at the focus of much recent debate (Doebeli and Dieckmann 2005; Doebeli et al. 2005; Gavrilets 2005; Polechová and Barton 2005; Waxman and Gavrilets 2005b). In this paper, we have studied a genetically simplified version of the model by Dieckmann and Doebeli (1999), where the evolution of assortative mating is governed by the relative fitness of homozygotes and heterozygotes. This simple criterion allowed us to gain a detailed overview of what can happen in a model of competitive speciation, along with a mecha- 

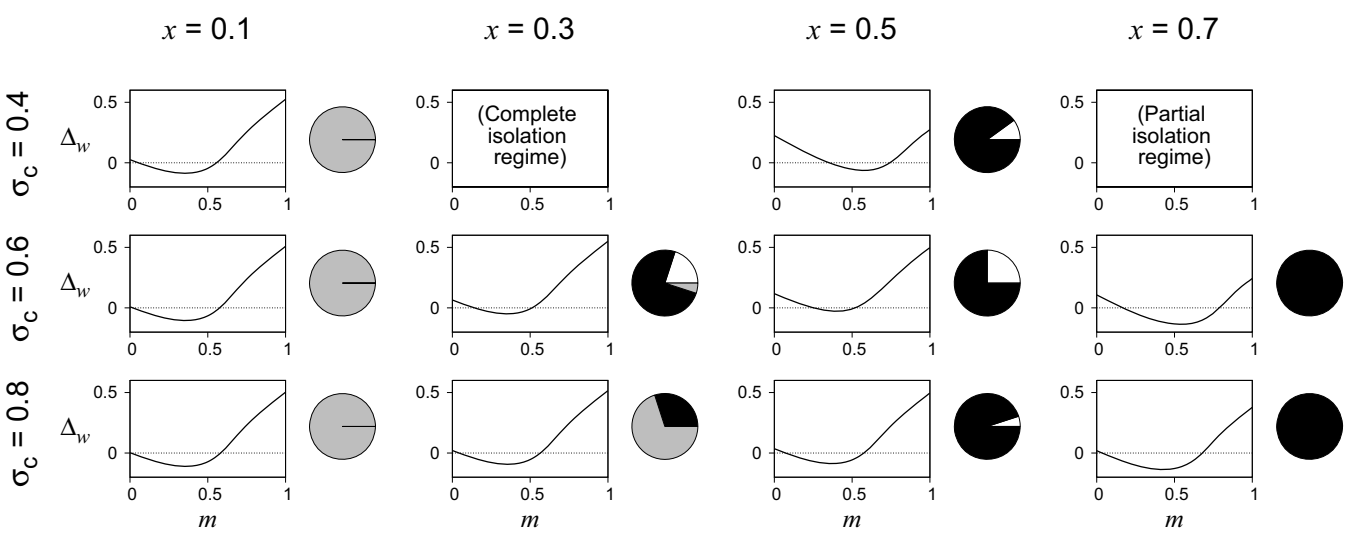

Figure 5: Alternative evolutionary outcomes in the $\mathrm{P} / \mathrm{C}$ regime (model 2), and fitness advantage of homozygotes, for various values of $x$ and $\sigma_{c}$. The rectangular panels show $\Delta_{W}=W_{\text {hom }}-W_{\text {het }}$ as a function of female choosiness $m$ (as in Fig. 2). The pie charts are based on 20 simulation runs each and show the probability that the polymorphism at the ecological locus is lost (grey), complete isolation is reached within 100,000 generations (white), or the population is "stuck" at the stable intermediate equilibrium for $m$ (black). Other parameters: $K_{0}=500$, mutation rate $u=4 \cdot 10^{-4}$, mutational step size is 0.1 .

nistic understanding of the relevant selection pressures. Our analysis leads to three major conclusions.

First, we find that evolution of complete reproductive isolation - and, as a consequence, sympatric speciation - is possible in a relevant area of parameter space under biologically realistic conditions. In the complete-isolation $(\mathrm{C})$ regime, speciation does not require any unusual assumptions about the strength of selection, nor does it depend on extreme initial conditions or unrealistically high mutation rates and mutational effects (Waxman and Gavrilets 2005a).

Second, we find that another frequent evolutionary outcome is partial reproductive isolation (see Doebeli 1996; Matessi et al. 2001). Partial isolation evolves if selection against intermediate phenotypes stops once they are sufficiently rare. It can be either locally or globally stable. At partial isolation, heterozygotes are still present in the population, but they are less frequent than at Hardy-Weinberg equilibrium. For most parameter combinations, the phenotypic distribution at the partial isolation equilibrium is still unimodal (results not shown). For parameters close to the domain of the complete isolation regime, however, the phenotypic distribution becomes bimodal, and isolation may be strong enough to imply, or eventually cause, speciation. We note that strong, but not complete isolation is also a common outcome of the Dieckmann and Doebeli (1999) model (see Fig. 3 in Dieckmann and Doebeli 1999, and Fig. 2 in Doebeli and Dieckmann 2003). In general, we suggest that the possibility of 


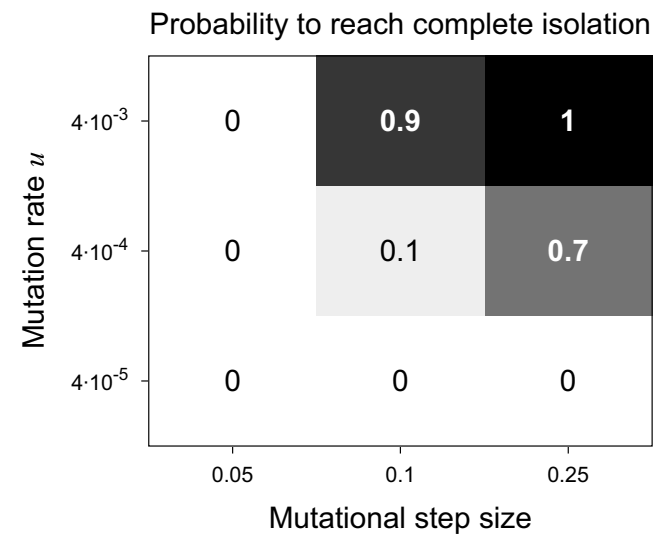

Figure 6: Probability to reach complete isolation in the $\mathrm{P} / \mathrm{C}$ regime (model 2) within 100,000 generations, depending on the mutation rate and the mutational step size. The number of steps between random mating and complete isolation is $1 /$ (step size) and ranges from 4 to 20 . The population was assumed to be at complete isolation when there were no heterozygotes left. Other parameters: $\sigma_{c}=0.4, x=0.5, K_{0}=500$. As can be seen from the corresponding panel in Figure 2, the width of the fitness valley that must crossed is about 0.4 .

partial isolation as a stable outcome of frequency-dependent disruptive selection deserves more attention than it currently receives. In particular, partial isolation between natural populations (e. g., Knudsen et al. 2006; Strecker 2006) does not necessarily imply incipient speciation (see also Bolnick 2006).

Finally, we find that whether competition leads to speciation or not depends on the combination of many parameters, both ecological and genetic. For example, for complete isolation to evolve in small steps, frequency-dependent selection due to competition must be neither too weak nor too strong, and speciation can be both facilitated or inhibited by sexual selection, the shape of the competition and mating functions, and the genetics of the mating trait. Therefore, conclusive quantitative predictions about the likelihood of various evolutionary outcomes cannot be made without good knowledge about the distribution of parameter values in nature. Simple comparisons of the size of various evolutionary regimes can be misleading. To emphasize this point, note that the hatched area corresponding to loss of polymorphism looks much more impressive in Figure 1c than in Figure 1d, but the differences between the two figures are brought about solely by a change in the parameters used to define the axis. Unfortunately, relevant data are difficult to obtain and largely absent to date. Given the multitude of parameters that jointly play a role, it may in fact be impossible to ever know all the relevant details for a specific empirical system. A more viable approach 
is to test statistical model predictions for the influence of various factors in a multi-species comparison.

\section{Natural and sexual selection}

In our model, evolution of assortative mating shows a remarkably complex equilibrium structure, which results from the interplay of natural and sexual selection. Natural selection includes stabilizing selection due to the dependence of carrying capacity on phenotype and frequency-dependent selection due to competition. In the model without sexual selection, evolution of complete reproductive isolation is possible if the resource supply for both homozygote classes is sufficiently high and their overlap in resource use is sufficiently low. If both conditions are satisfied, the two evolving species can occupy two distinct ecological niches. If, however, the competition function is too narrow, a third niche at an intermediate phenotype opens up that is filled by the heterozygotes, thus preventing complete isolation. The size of this third niche determines the "optimal" ratio of heterozygotes to homozygotes in the population, and female choosiness then evolves to such a value that this optimal ratio is produced. If the niche for heterozygotes gets sufficiently large, natural selection stabilizes random mating. As long as natural selection is disruptive $(c>k)$, it favors a unique evolutionary equilibrium, and the ecological polymorphism is always stable. We, therefore, find three evolutionary regimes: complete-isolation $(\mathrm{C})$, partial-isolation $(\mathrm{P})$, and randommating $(\mathrm{R})$.

Sexual selection against rare males can either enhance or oppose the effects of natural selection. It favors heterozygotes when they are common and disfavors them when they are rare. Thus, sexual selection promotes speciation if female choosiness is already high, but impedes it as long as choosiness is low. This positive frequency-dependence can cause bistability of the evolutionary equilibria: In the $\mathrm{R} / \mathrm{C}$ regime, natural selection favors random mating, but sexual selection can maintain complete isolation once it has been established. In the $\mathrm{P} / \mathrm{C}$ regime, natural selection favors partial or complete isolation, but sexual selection favors heterozygotes at low levels of choosiness and creates a barrier against the evolution of stronger assortative mating. An intermediate equilibrium is reached if natural and sexual selection balance. This equilibrium has previously been observed by Doebeli (1996) and Matessi et al. (2001), but these authors did not provide a mechanistic explanation. As a consequence of the 
above barrier, sexual selection does not increase the area where complete isolation can evolve from random mating in small steps, although it usually increases the area in parameter space where complete isolation is locally stable (Fig. 1). In addition, sexual selection can lead to the loss of the ecological polymorphism, as previously described by Gourbière (2004), Kirkpatrick and Nuismer (2004), Bürger et al. (2006), and Bürger and Schneider (2006). This is most relevant for small populations where frequency-dependent selection due to competition is weak and a monomorphic equilibrium is almost always reached (see Figure 5).

In summary, there are three mechanisms that can prevent the evolution of complete reproductive isolation, even if selection is disruptive: (i) Natural selection can stabilize a sufficiently small proportion of heterozygotes in a third niche, (ii) sexual selection can favor heterozygotes while they are common, and (iii) sexual selection can cause the loss of the ecological polymorphism. The distinction between the latter two mechanisms has not always been made clear in the past. For example, Bürger and Schneider (2006) described the loss of polymorphism as a consequence of the evolution to an intermediate optimum for female choosiness. However, our analysis shows that these phenomena are based on different mechanisms. An infinite population that takes small mutational steps will always evolve to partial isolation without losing the polymorphism (see Figure 3). A finite population, however, can (and often will) lose the polymorphism if it moves past the stable intermediate equilibrium by drift (Figure 5).

\section{Discussion of the modelling approach}

Our approach in this study was to analyze a simplified version of the model by Dieckmann and Doebeli (1999). This approach allowed us to (a) derive a simple invasion criterion that yielded general analytical results and enabled us to exhaustively analyze the model in a seven-dimensional parameter space, (b) to gain a detailed and intuitive understanding of the interaction between the various selective forces, and (c) to unify, in a single model, a large number of phenomena that have previously been studied or described only in separate studies. The latter include (i) the role of natural versus sexual selection (see Gourbière 2004; Kirkpatrick and Nuismer 2004), (ii) conditions for the maintenance or loss of the ecological polymorphism (see Kirkpatrick and Nuismer 2004; Bürger and Schneider 2006; Bürger et al. 2006), (iii) potential evolutionary stability of incomplete isolation (Doebeli 
1996; Matessi et al. 2001), and (iv) the importance of ecological niches and the resulting nonlinear relationship between niche width and the likelihood of speciation (Seger 1985; Dieckmann and Doebeli 1999; Gourbière 2004; Bolnick 2006; Bürger et al. 2006).

In addition, our approach allowed us to analyze fitness functions of different shape. Many previous models have used Gaussian functions like those in equations (1), (2) and (4), but this is more for mathematical than biological reasons. Indeed, several authors have pointed out that the Gaussian version of the Roughgarden (1972) model can show non-generic behavior (e. g., Sasaki and Ellner 1995; Sasaki 1997; Gyllenberg and Meszéna 2005; Polechová and Barton 2005; Doebeli et al. 2007). Therefore, it is important to know how the likelihood of speciation depends on the Gaussian assumption. In our model, the shape of the competition function determines which regimes are possible. For example, in the absence of sexual selection, complete isolation is evolutionarily stable if and only if the competition function is convex (i. e., if the strength of competition decreases more than linearly with phenotypic distance). Matessi et al. (2001) used a quadratic competition function, which can be seen as a weakselection approximation of more complex functions. Under this approximation, heterozygotes always have the highest death rate and (for $c>k$ ) complete isolation is always locally stable. Therefore, Matessi et al. (2001) found only two of our five regimes, complete isolation and $\mathrm{P} / \mathrm{C}$. It is in the regions where the weak-selection approximation is not valid that we find the other three regimes. It is worth pointing out that in the absence of sexual selection, neither the quadratic nor the Gaussian function captures the full complexity of the model (see Fig. A1a). Indeed, even for weak selection, where the two functions approximate each other, the quadratic function does not allow for the partial-isolation regime, whereas the Gaussian function does not allow for the $\mathrm{R} / \mathrm{C}$ regime (at $c<k$ ). This shows that apparently small details can have a qualitative influence on the behavior of the model, and that it is important to study the complete parameter space. Remarkably, potential asymmetries of the competition and the carrying capacity function seem to have a relatively small effect on the regime pattern (see Appendix 5).

The key to our analysis is the invasion criterion, according to which choosiness increases evolutionarily whenever homozygotes have a higher fitness than heterozygotes. This criterion can be shown to hold true for different modes of sexual selection, and the approach can be readily extended, for example, to cases in which also males are choosy. However, the criterion 
is not valid if there is a direct cost of choosiness. In this case, the fitness of a mutant depends not only on the mating strategies in the resident population but also on the strategy of the mutant itself. Costs of choosiness can arise, for example, if choosy females must spend more energy during mate choice or if they are less likely to find a suitable mate eventually. Considerable debate has focused on the question whether costs of choosiness are likely to prevent competitive speciation (Doebeli and Dieckmann 2003; Bolnick 2004b; Doebeli and Dieckmann 2005; Gavrilets 2005; Waxman and Gavrilets 2005a,b). Some recent models indicate that moderate costs of choosiness are not necessarily detrimental for the possibility of speciation (Doebeli and Dieckmann 2003, 2005; Schneider and Bürger 2005; Bürger and Schneider 2006; Doebeli et al. 2007). Nevertheless, including costs of choosiness would be an important extension of our model. One way to do so might be by a direct derivation of the invasion fitness in a modified model along the lines of Appendix 2.

The key simplification in our model is the assumption that the ecological trait is determined by a single locus with two alleles. How general are our results with regard to this genetic architecture? On the one hand, it seems reasonable to expect that the five regimes, which we have described here, are generic also for other genetic architectures, because the interplay of natural and sexual selection should be qualitatively independent of genetic details. This intuition is supported by the observation that a behavior similar to the $\mathrm{P} / \mathrm{C}$ regime has also been found in a multilocus model by Doebeli (1996). Furthermore, evolution of strong reproductive isolation (leading to a multimodal phenotype distribution) has been demonstrated in a number of models with very different genetic assumptions, spanning the whole range from one-locus models (Matessi et al. 2001, this study), to multilocus models (Doebeli 1996; Dieckmann and Doebeli 1999; Bolnick 2006), and finally to quantitative genetic models that are based on an effectively infinite number of loci (Doebeli et al. 2007).

On the other hand, the one-locus assumption has the obvious consequence that intermediate phenotypes can only exist as heterozygotes. Therefore, whenever more than two phenotypes can potentially coexist (i. e., whenever there are more than two ecological niches), natural selection tends to move the population toward partial isolation or random mating. In a model with a different genetic architecture, the evolution of assortative mating might instead lead to more than two reproductively isolated species (Bolnick 2006; Bürger et al. 2006). In addition, monomorphic equilibria could play a more prominent role if they exist very close to the fitness optimum (Bürger et al. 2006). The one-locus assumption is also essential for 
the behavior of the model in the parameter range where stabilizing selection dominates over competition ( $c<k$, or $\sigma_{c}>\sigma_{K}$ in the Gaussian case). In a multilocus model, natural selection will then usually remove genetic variation from the ecological locus and keep at most one locus polymorphic (Christiansen and Loeschcke 1980; Spichtig and Kawecki 2004; Bürger 2005). In a single-locus model, however, this transition is not clearly visible, since heterozygote advantage guarantees the polymorphism of the locus even for $c<k$.

A related question concerns the genetic architecture of female choosiness. Part of the controversy about sympatric speciation has centered on how the likelihood of speciation depends on the choice of mutational parameters for the loci determining assortative mating (Doebeli and Dieckmann 2005; Waxman and Gavrilets 2005b). In our simulations, we assumed that choosiness is based on a single additive locus with a continuum of possible alleles, and we have varied the mutation rate and mutational step size. Our results lead to two conclusions: First, in the complete-isolation regime, where speciation is possible from random mating in small steps, the evolutionary outcome should be independent of genetic details. In particular, for a given mean mutational step size, the time to reach complete isolation depends only on the product of mutation rate and population size. Second, in the bistable $\mathrm{P} / \mathrm{C}$ regime, the likelihood of speciation does, indeed, depend on the genetic architecture of the mating trait. In populations evolving in small mutational steps, the intermediate equilibrium for female choosiness forms a "barrier" against the evolution of complete isolation. However, our simulations suggest that a population can "jump" over this barrier if mutation rates and mutational effects are sufficiently high (Fig. 6). Probably, this is most likely in small populations under the influence of genetic drift.

For more general genetic architectures, the likelihood of jumping the intermediate equilibrium is still an open question. For example, in the infinitesimal model of Doebeli et al. (2007), degrees of female choosiness are assumed to be normally distributed in the population, and the tail of this distribution contains individuals with very high choosiness, which might initiate the jump to higher degrees of isolation. A similar conclusion appears to hold in multilocus models, in which recombination can create an analogous tail (Dieckmann and Doebeli 1999). We, therefore, propose the following hypothesis: Speciation in the $\mathrm{P} / \mathrm{C}$ regime is possible if choosiness has either a simple genetic basis, so that complete isolation can be reached in a small number of mutational steps, or if it is determined by a large number of loci that help maintain a high degree of genetic variation for choosiness. 
Finally, another important assumption of our model is that the allelic effect of the ecological locus as well as the niche shape are constant. In principle, they might also be subject to selection (Kisdi and Geritz 1999; Geritz and Kisdi 2000; Ackermann and Doebeli 2004; Kopp and Hermisson 2006; van Doorn and Dieckmann 2006; Schneider 2007). The joint evolution of assortative mating with genetic architecture and / or individual specialization (Ackermann and Doebeli 2004; Rueffler et al. 2006) is an interesting avenue for future studies.

Acknowledgements. PSP, MK, and JH where supported by an Emmy-Noether grant from the Deutsche Forschungsgemeinschaft (DFG) to JH. PSP acknowledges a grant from the Dutch Science Foundation (NWO) for participation in the IIASA Young Scientists Summer Program. GM was supported by grants T049689 and TS049885 from the Hungarian Scientific Research Fund (OTKA). UD acknowledges financial support from the Vienna Science and Technology Fund (WWTF). GM thanks for discussions with Freddy Christiansen, with whom he started to think about the issues presented in this paper. We also thank Jim Fry, Mark Kirkpatrick, Sally Otto, Agnes Rettelbach, and Kristan Schneider for their valuable comments on earlier versions of the manuscript (in particular, Sally Otto challenged us to extend our analysis to the asymmetric model). Finally, we thank Mark Kirkpatrick and Sally Otto for sharing their unpublished manuscripts. 


\section{Appendices}

\section{Appendix 1: Equations for the one-locus, two-allele model}

In this Appendix, we spell out the equations for the one-locus, two-allele model (assuming all females are equally choosy). The male mating rates (see eq. 5b) are given by

$$
\begin{aligned}
\phi_{\text {male hom }}^{ \pm} & =N_{\mathrm{hom}}^{ \pm} M_{\mathrm{hom}}^{ \pm}+(1-m) N_{\mathrm{het}} M_{\mathrm{het}}+\left(1-m^{\prime}\right) N_{\mathrm{hom}}^{\mp} M_{\mathrm{hom}}^{\mp} \\
\phi_{\text {male, het }} & =(1-m) N_{\mathrm{hom}}^{+} M_{\mathrm{hom}}^{+}+N_{\mathrm{het}} M_{\mathrm{het}}+(1-m) N_{\mathrm{hom}}^{-} M_{\mathrm{hom}}^{-}
\end{aligned}
$$

and the female mating rates (eq. 5a) by

$$
\begin{aligned}
\phi_{\text {female, hom }}^{ \pm} & =N_{\mathrm{hom}}^{ \pm} M_{\mathrm{hom}}^{ \pm}+(1-m) N_{\mathrm{het}} M_{\mathrm{hom}}^{ \pm}+\left(1-m^{\prime}\right) N_{\mathrm{hom}}^{\mp} M_{\mathrm{hom}}^{ \pm} \\
\phi_{\text {female, het }} & =(1-m) N_{\mathrm{hom}}^{+} M_{\mathrm{het}}+N_{\mathrm{het}} M_{\mathrm{het}}+(1-m) N_{\mathrm{hom}}^{-} M_{\mathrm{het}} .
\end{aligned}
$$

In the model with sexual selection, $\phi_{\text {female }}=1$ for all genotypes, which is satisfied by the mating activity factors

$$
\begin{aligned}
M_{\text {hom }}^{ \pm} & =\left[N_{\text {hom }}^{ \pm}+(1-m) N_{\text {het }}+\left(1-m^{\prime}\right) N_{\text {hom }}^{\mp}\right]^{-1}, \\
M_{\text {het }} & =\left[(1-m) N_{\text {hom }}^{+}+N_{\text {het }}+(1-m) N_{\text {hom }}^{-}\right]^{-1} .
\end{aligned}
$$

In the model without sexual selection, $\phi=1 / 2\left(\phi_{\text {male }}+\phi_{\text {female }}\right)=1$ for all genotypes. The activity factors for a population that is monomorphic at the mating locus can be derived from the linear equation system $\phi_{\text {hom }}=\phi_{\text {het }}=1$. For the symmetric case, where $N_{\text {hom }}^{+}=$ $N_{\text {hom }}^{-}=N_{\text {hom }}$, we find from equations (A1) and (A2),

$$
\begin{aligned}
\phi_{\text {hom }} & =\left(2-m^{\prime}\right) N_{\text {hom }} M_{\text {hom }}+(1-m) N_{\text {het }} \frac{M_{\text {hom }}+M_{\text {het }}}{2}=1 \\
\phi_{\text {het }} & =N_{\text {het }} M_{\text {het }}+(1-m) N_{\text {hom }}\left(M_{\text {hom }}+M_{\text {het }}\right)=1,
\end{aligned}
$$

yielding 


$$
\begin{aligned}
M_{\text {hom }} & =\frac{n+(1-n / 2)(1-m)}{N_{\text {hom }}\left[\left(2-m^{\prime}\right)(n+1-m)+n^{2}(1-m) / 2\right]}, \\
M_{\text {het }} & =\frac{2-m^{\prime}-(1-n / 2)(1-m)}{N_{\text {hom }}\left[\left(2-m^{\prime}\right)(n+1-m)+n^{2}(1-m) / 2\right]} .
\end{aligned}
$$

For a general polymorphic population, the activity factors follow from the additional condition that female mating rate should not depend on the degree of choosiness, but only on the ecological genotype (see Appendix 2 for the case of invasion fitness).

The genotype-specific birth rates (eq. 6) for both models are

$$
\begin{aligned}
B_{\text {hom }}^{ \pm}= & N_{\text {hom }}^{ \pm}\left(N_{\text {hom }}^{ \pm}+\frac{(1-m) N_{\text {het }}}{2}\right) M_{\text {hom }}^{ \pm}+ \\
& N_{\text {het }}\left(\frac{(1-m) N_{\text {hom }}^{ \pm}}{2}+\frac{N_{\text {het }}}{4}\right) M_{\text {het }}, \\
B_{\text {het }}= & N_{\text {hom }}^{+}\left(\frac{(1-m) N_{\text {het }}}{2}+\left(1-m^{\prime}\right) N_{\text {hom }}^{-}\right) M_{\text {hom }}^{+}+\frac{N_{\text {het }}}{2}+ \\
& N_{\text {hom }}^{-}\left(\left(1-m^{\prime}\right) N_{\text {hom }}^{+}+\frac{(1-m) N_{\text {het }}}{2}\right) M_{\text {hom }}^{-} .
\end{aligned}
$$

Furthermore, the effective population sizes with respect to competition (see eq. 3) are given by

$$
\begin{aligned}
C_{\mathrm{hom}}^{ \pm} & =N_{\mathrm{hom}}^{ \pm}+\left(1-c^{ \pm}\right) N_{\mathrm{het}}+\left(1-c^{\prime}\right) N_{\mathrm{hom}}^{\mp}, \\
C_{\text {het }} & =\left(1-c^{+}\right) N_{\mathrm{hom}}^{+}+N_{\text {het }}+\left(1-c^{-}\right) N_{\text {hom }}^{-},
\end{aligned}
$$

and the death rates (see eq. 8) by

$$
d_{\mathrm{hom}}^{ \pm}=\frac{C_{\mathrm{hom}}^{ \pm}}{K_{\mathrm{hom}}^{ \pm}}, \quad d_{\mathrm{het}}=\frac{C_{\mathrm{het}}}{K_{\mathrm{het}}} .
$$

With these definitions, the fitness functions of the three ecological genotypes (according to eq. 9) can be written as

$$
\begin{aligned}
W_{\text {hom }}^{ \pm} & =\frac{1}{2}\left(\phi_{\text {female, hom }}^{ \pm}+\phi_{\text {male, hom }}^{ \pm}\right)-d_{\text {hom }}^{ \pm}, \\
W_{\text {het }} & =\frac{1}{2}\left(\phi_{\text {female, het }}+\phi_{\text {male, het }}\right)-d_{\text {het }} .
\end{aligned}
$$




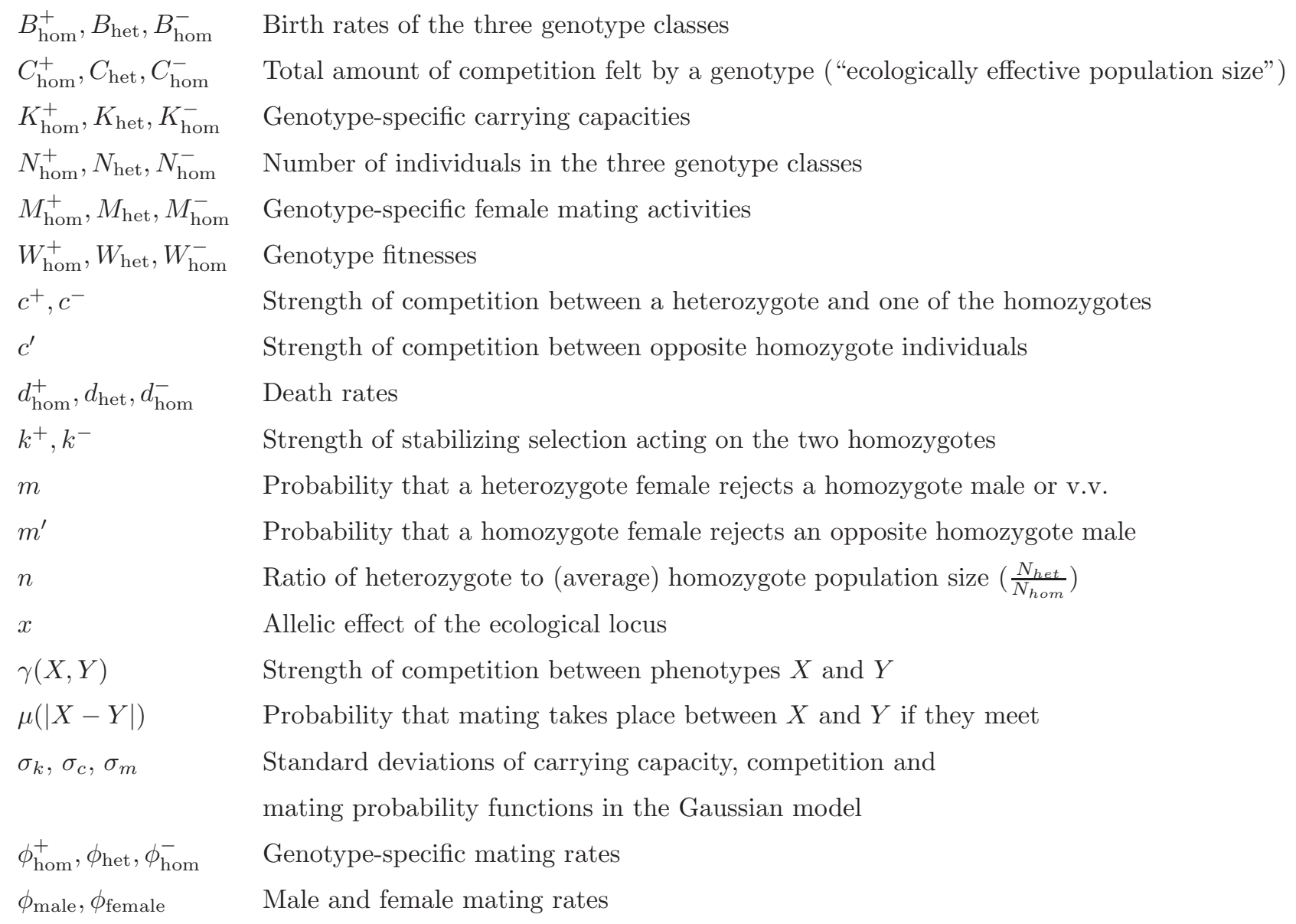

Table A1: Major model parameters. Averages over the two homozygote classes are expressed by corresponding parameters without a \pm index, e. g., $N_{\text {hom }}=\left(N_{\text {hom }}^{+}+N_{\text {hom }}^{-}\right) / 2$.

Finally, the dynamics of genotype frequencies (see eq. 7) are given by

$$
\begin{gathered}
\dot{N}_{\text {hom }}^{ \pm}=B_{\text {hom }}^{ \pm}-N_{\text {hom }}^{ \pm} d_{\text {hom }}^{ \pm} \\
\dot{N}_{\text {het }}=B_{\text {het }}-N_{\text {het }} d_{\text {het }}
\end{gathered}
$$

\section{Appendix 2: Invasion analysis}

Here, we prove for the general asymmetric model that a mutant with stronger female choosiness (higher $m$ and $m^{\prime}$ ) than the resident will be able to invade the population at the polymorphic equilibrium of the resident if and only if $W_{\text {hom }}>W_{\text {het }}$. Vice versa, a mutant with weaker assortative mating will invade if and only if $W_{\text {hom }}<W_{\text {het }}$. Rare assortment modifiers are neutral (i.e., have zero invasion fitness) if and only if $W_{\mathrm{hom}}^{+}=W_{\text {het }}=W_{\text {hom }}^{-}=0$. 
Note first that, at equilibrium, $W_{\text {hom }}^{+}$and $W_{\text {hom }}^{-}$always have the same sign and $W_{\text {het }}$ has the opposite sign. This is a direct consequence of the equilibrium condition for + and - alleles in the population,

$$
\begin{aligned}
& 2 N_{\text {hom }}^{+} W_{\text {hom }}^{+}+W_{\text {het }} N_{\text {het }}=0, \\
& 2 N_{\text {hom }}^{-} W_{\text {hom }}^{-}+W_{\text {het }} N_{\text {het }}=0
\end{aligned}
$$

which implies $N_{\text {hom }}^{+} W_{\text {hom }}^{+}=N_{\text {hom }}^{-} W_{\text {hom }}^{-}$.

Consider now a resident population with choosiness $m$ and $m^{\prime}$ and a rare mutant allele at the choosiness locus that leads to larger (or smaller) values $\tilde{m}$ and $\tilde{m}^{\prime}$. As the mutant allele is rare, an individual carries at most one copy, and matings among mutants can be ignored. For simplicity, we will first treat the case of free recombination between the ecological locus and the mating locus (recombination rate $\rho=1 / 2$ ). We can then identify the two types of double heterozygotes and denote their number by $\nu_{\text {het }}$. Similarly, $\nu_{\text {hom }}^{+}$and $\nu_{\text {hom }}^{-}$are the numbers of ecological $(+/+)$ and $(-/-)$ homozygotes that carry the mutant choosiness allele.

The dynamics of the mutant population is then governed by the following equations:

$$
\begin{aligned}
& \dot{\nu}_{\text {het }}=\tilde{B}_{\text {het } \leftarrow \text { female }}+\tilde{B}_{\text {het } \leftarrow \text { male }}-\nu_{\text {het }} d_{\text {het }} \\
& \dot{\nu}_{\text {hom }}^{ \pm}=\tilde{B}_{\text {hom } \leftarrow \text { female }}^{ \pm}+\tilde{B}_{\text {hom } \leftarrow \text { male }}^{ \pm}-\nu_{\text {hom }}^{ \pm} d_{\text {hom }}^{ \pm}
\end{aligned}
$$

where the dot denotes the time derivative. Here, for example, $\tilde{B}_{\text {het } \leftarrow \text { female }}$ is the birth rate of heterozygote mutants that have inherited the mutant allele from their mother. Note that for a female parent, but not for a male parent, these rates depend on the mutant choosiness. Explicitly, 


$$
\begin{aligned}
& \tilde{B}_{\text {het } \leftarrow \text { female }}=\frac{1}{4} N_{\text {het }} \nu_{\text {het }} \tilde{M}_{\text {het }}+\frac{1}{2}\left(1-\tilde{m}^{\prime}\right)\left(N_{\text {hom }}^{-} \nu_{\text {hom }}^{+} \tilde{M}_{\text {hom }}^{+}+N_{\text {hom }}^{+} \nu_{\text {hom }}^{-} \tilde{M}_{\text {hom }}^{-}\right) \\
& +\frac{1}{4}(1-\tilde{m})\left(N_{\text {het }}\left(\nu_{\text {hom }}^{+} \tilde{M}_{\text {hom }}^{+}+\nu_{\text {hom }}^{-} \tilde{M}_{\text {hom }}^{-}\right)+\left(N_{\text {hom }}^{+}+N_{\text {hom }}^{-}\right) \nu_{\text {het }} \tilde{M}_{\text {het }}\right), \\
& \tilde{B}_{\text {het } \leftarrow \text { male }}=\frac{1}{4} N_{\text {het }} \nu_{\text {het }} M_{\text {het }}+\frac{1}{2}\left(1-m^{\prime}\right)\left(N_{\text {hom }}^{-} \nu_{\text {hom }}^{+} M_{\text {hom }}^{-}+N_{\text {hom }}^{+} \nu_{\text {hom }}^{-} M_{\text {hom }}^{+}\right) \\
& +\frac{1}{4}(1-m)\left(N_{\text {het }}\left(\nu_{\text {hom }}^{+}+\nu_{\text {hom }}^{-}\right) M_{\text {het }}+\left(N_{\text {hom }}^{+} M_{\text {hom }}^{+}+N_{\text {hom }}^{-} M_{\text {hom }}^{-}\right) \nu_{\text {het }}\right), \\
& \tilde{B}_{\text {hom } \leftarrow \text { female }}^{ \pm}=\frac{1}{8} N_{\text {het }} \nu_{\text {het }} \tilde{M}_{\text {het }}+\frac{1}{4}(1-\tilde{m}) N_{\text {hom }}^{ \pm} \nu_{\text {het }} \tilde{M}_{\text {het }} \\
& +\frac{1}{2} N_{\mathrm{hom}}^{ \pm} \nu_{\mathrm{hom}}^{ \pm} \tilde{M}_{\mathrm{hom}}^{ \pm}+\frac{1}{4}(1-\tilde{m}) N_{\mathrm{het}} \nu_{\mathrm{hom}}^{ \pm} \tilde{M}_{\mathrm{hom}}^{ \pm}, \\
& \tilde{B}_{\text {hom } \leftarrow \text { male }}^{ \pm}=\frac{1}{8} N_{\text {het }} \nu_{\text {het }} M_{\text {het }}+\frac{1}{4}(1-m) N_{\text {hom }}^{ \pm} \nu_{\text {het }} M_{\text {hom }}^{ \pm} \\
& +\frac{1}{2} N_{\mathrm{hom}}^{ \pm} \nu_{\mathrm{hom}}^{ \pm} M_{\mathrm{hom}}^{ \pm}+\frac{1}{4}(1-m) N_{\mathrm{het}} \nu_{\mathrm{hom}}^{ \pm} M_{\mathrm{het}} .
\end{aligned}
$$

Here, it was taken into account that only half of the offspring of a choosiness heterozygote carry the mutant choosiness allele. $M$ and $\tilde{M}$ denote the activity factors of female residents and mutants, respectively. We can write the mutant dynamics in matrix form, $\boldsymbol{v}=\boldsymbol{A} \boldsymbol{v}$,

$$
\left(\begin{array}{c}
\dot{\nu}_{\text {hom }}^{+} \\
\dot{\nu}_{\text {het }} \\
\dot{\nu}_{\text {hom }}^{-}
\end{array}\right)=\left(\begin{array}{lll}
a_{11} & a_{12} & a_{13} \\
a_{21} & a_{22} & a_{23} \\
a_{31} & a_{32} & a_{33}
\end{array}\right) \cdot\left(\begin{array}{c}
\nu_{\text {hom }}^{+} \\
\nu_{\text {het }} \\
\nu_{\text {hom }}^{-}
\end{array}\right) .
$$

The matrix depends on the mating genotypes of the residents and the mutants, $\boldsymbol{A}=\boldsymbol{A}_{\boldsymbol{m}, \tilde{\boldsymbol{m}}}$, where $\boldsymbol{m}=\left(m, m^{\prime}\right)$ denotes the vector of mating parameters. For the following, it will be convenient to express the elements $a_{i j}$ of $\boldsymbol{A}_{\boldsymbol{m}, \tilde{\boldsymbol{m}}}$ (partly) in terms of the mating rates $\phi_{\text {hom }}^{ \pm}=\left(\phi_{\text {male, hom }}^{ \pm}+\phi_{\text {female, hom }}^{ \pm}\right) / 2$ and $\phi_{\text {het }}=\left(\phi_{\text {male, het }}+\phi_{\text {female, het }}\right) / 2$. We can then use the fact that the mating rates of invading mutants are equal to the equilibrium mating rates of the resident. For the male mating rates $\phi_{\text {male }}$, this is simply because mutant females are rare. For the female mating rates, it is a consequence of our assumption that changes in choosiness come without direct costs. In particular, the mating genotype does not affect the female mating rate (we have $\phi_{\text {female }}=1-\phi_{\text {male }}=$ const. for model 1 and $\phi_{\text {female }}=1$ for model 2 ). Explicitly, we obtain 


$$
\boldsymbol{A}_{\boldsymbol{m}, \tilde{\boldsymbol{m}}}=\left(\begin{array}{ccc}
\frac{1}{2} \phi_{\mathrm{hom}}^{+}-d_{\mathrm{hom}}^{+}+Q^{+} & \frac{1}{4} \phi_{\mathrm{het}}+R & 0 \\
\frac{1}{2} \phi_{\mathrm{hom}}^{+}-Q^{+} & \frac{1}{2} \phi_{\mathrm{het}}-d_{\mathrm{het}} & \frac{1}{2} \phi_{\mathrm{hom}}^{-}-Q^{-} \\
0 & \frac{1}{4} \phi_{\mathrm{het}}-R & \frac{1}{2} \phi_{\mathrm{hom}}^{-}-d_{\mathrm{hom}}^{-}+Q^{-}
\end{array}\right)
$$

with

$$
\begin{aligned}
Q^{ \pm}(\boldsymbol{m}, \tilde{\boldsymbol{m}}) & =\frac{1}{4}\left(\tilde{M}_{\mathrm{hom}}^{ \pm}\left(N_{\mathrm{hom}}^{ \pm}-\left(1-\tilde{m}^{\prime}\right) N_{\mathrm{hom}}^{\mp}\right)+\left[M_{\mathrm{hom}}^{ \pm} N_{\mathrm{hom}}^{ \pm}-\left(1-m^{\prime}\right) M_{\mathrm{hom}}^{\mp} N_{\mathrm{hom}}^{\mp}\right]\right) \\
R(\boldsymbol{m}, \tilde{\boldsymbol{m}}) & =\frac{1}{8}\left(\tilde{M}_{\mathrm{het}}(1-\tilde{m})\left(N_{\mathrm{hom}}^{+}-N_{\mathrm{hom}}^{-}\right)+(1-m)\left(N_{\mathrm{hom}}^{+} M_{\mathrm{hom}}^{+}-N_{\mathrm{hom}}^{-} M_{\mathrm{hom}}^{-}\right)\right) .
\end{aligned}
$$

In both $R$ and $Q$, the first and second terms correspond, respectively, to cases where the mutant allele is carried by the female or the male partner. Before we proceed, we note several elementary facts:

1. Let $\mathbb{I}$ be the identity matrix. Then $\hat{\boldsymbol{A}}:=\boldsymbol{A}+\left(d_{\mathrm{hom}}^{+}+d_{\mathrm{het}}+d_{\mathrm{hom}}^{-}\right) \mathbb{I}$ is positive semi-definite (and its square is strictly positive). According to the Perron-Frobenius theorem, $\hat{\boldsymbol{A}}$ has a unique dominant eigenvalue and the corresponding left and right eigenvectors have strictly positive entries. Since $\boldsymbol{A}$ and $\hat{\boldsymbol{A}}$ have the same eigenvectors, the same is true for $\boldsymbol{A}$.

2. The columns of $\boldsymbol{A}_{\boldsymbol{m}, \tilde{\boldsymbol{m}}}$ sum up to the genotype fitnesses, i.e., $W_{\mathrm{hom}}^{+}=a_{11}+a_{21}+a_{31}$, $W_{\text {het }}=a_{12}+a_{22}+a_{32}$, and $W_{\text {hom }}^{-}=a_{13}+a_{23}+a_{33}$.

3. For $\boldsymbol{m}=\tilde{\boldsymbol{m}}$, the matrix $\boldsymbol{A}_{\boldsymbol{m}, \boldsymbol{m}}$ reproduces the population dynamics of the resident population (Eqs. A10). The dominant eigenvalue of this "resident matrix" $\boldsymbol{A}_{\boldsymbol{m}, \boldsymbol{m}}$ is 0 and the corresponding right eigenvector is proportional to the equilibrium distribution, $\left(N_{\text {hom }}^{+}, N_{\text {het }}, N_{\text {hom }}^{-}\right)$.

For the next step, we consider the leading left eigenvector of the resident matrix $\boldsymbol{A}_{\boldsymbol{m}, \boldsymbol{m}}$, which describes the "reproductive values" of the three ecological genotypes (cf. Caswell 2000, p. $92)$. Denote this eigenvector by $\boldsymbol{v}:=\left(v^{+}, v_{\text {het }}, v^{-}\right)$. Without loss of generality, we can set $v_{\text {het }}=1$. Then 


$$
\begin{aligned}
& v^{+}=-\frac{a_{21}}{a_{11}}=-\frac{a_{21}}{W_{\mathrm{hom}}^{+}-a_{21}}=\frac{2 N_{\mathrm{hom}}^{+} a_{21}}{2 N_{\mathrm{hom}}^{+} a_{21}+W_{\mathrm{het}} N_{\mathrm{het}}}, \\
& v^{-}=-\frac{a_{23}}{a_{33}}=-\frac{a_{23}}{W_{\mathrm{hom}}^{-}-a_{23}}=\frac{2 N_{\mathrm{hom}}^{-} a_{23}}{2 N_{\mathrm{hom}}^{-} a_{23}+W_{\mathrm{het}} N_{\mathrm{het}}},
\end{aligned}
$$

where we use (A11). Since $W_{\text {hom }}^{ \pm} \gtrless 0 \Leftrightarrow W_{\text {het }} \lessgtr 0$, we conclude that

$$
v^{ \pm} \gtrless v_{\text {het }} \Leftrightarrow W_{\text {hom }}^{ \pm} \gtrless W_{\text {het }} .
$$

To establish the rank order of $v^{+}$and $v^{-}$, we use the explicit expressions for $a_{21}$ and $a_{23}$ (eq. A19) to obtain

$$
N_{\text {hom }}^{+} a_{21} \gtrless N_{\text {hom }}^{-} a_{23} \Leftrightarrow N_{\text {hom }}^{+}\left(M_{\text {hom }}^{+}+M_{\text {het }}\right) \gtrless N_{\text {hom }}^{-}\left(M_{\text {hom }}^{-}+M_{\text {het }}\right) \Leftrightarrow N_{\text {hom }}^{+} \gtrless N_{\text {hom }}^{-},
$$

where we use the fact that $N_{\text {hom }}^{+} M_{\text {hom }}^{+} \leq N_{\text {hom }}^{-} M_{\text {hom }}^{-} \Leftrightarrow N_{\text {hom }}^{+} \leq N_{\text {hom }}^{-}$in both of our models. Using $N_{\text {hom }}^{+} W_{\text {hom }}^{+}=N_{\text {hom }}^{-} W_{\text {hom }}^{-}$,

$$
\frac{d}{d x} \frac{x}{x+W_{\text {het }} N_{\text {het }}}=\frac{W_{\text {het }} N_{\text {het }}}{\left(x+W_{\text {het }} N_{\text {het }}\right)^{2}},
$$

and the fact that the sign of $W_{\text {hom }}^{ \pm}$is opposite to the sign of $W_{\text {het }}$, we conclude that

$$
v^{+} \gtrless v^{-} \Leftrightarrow W_{\text {hom }}^{+} \gtrless W_{\text {hom }}^{-}
$$

Denote, now, the dominant eigenvalue of the full mutant matrix $\boldsymbol{A}_{\boldsymbol{m}, \tilde{\boldsymbol{m}}}$ as $\lambda(\boldsymbol{m}, \tilde{\boldsymbol{m}})$. In the terminology of adaptive dynamics theory (Metz et al. 1992; Dieckmann and Law 1996; Geritz et al. 1998), $\lambda$ is the invasion fitness. The mutant can invade the resident whenever $\lambda>0$. Let $\boldsymbol{w}:=\left(w^{+}, w_{\text {het }}, w^{-}\right)$be the leading right eigenvector of the mutant matrix $\boldsymbol{A}_{\boldsymbol{m}, \tilde{m}}$ (whereas $v$ is defined as the leading left eigenvector of the resident matrix $\boldsymbol{A}_{\boldsymbol{m}, \boldsymbol{m}}$ ). We can then express $\lambda$ as

$$
\begin{aligned}
\lambda(\boldsymbol{m}, \tilde{\boldsymbol{m}})= & \frac{\boldsymbol{v} \boldsymbol{A}_{\boldsymbol{m}, \tilde{\boldsymbol{m}}} \boldsymbol{w}}{\boldsymbol{v} \boldsymbol{w}}=\frac{\boldsymbol{v}\left(\boldsymbol{A}_{\boldsymbol{m}, \tilde{\boldsymbol{m}}}-\boldsymbol{A}_{\boldsymbol{m}, \boldsymbol{m}}\right) \boldsymbol{w}}{\boldsymbol{v} \boldsymbol{w}} \\
= & (\boldsymbol{v} \boldsymbol{w})^{-1}\left(w^{+}\left(v^{+}-v_{\mathrm{het}}\right) \Delta Q^{+}(\boldsymbol{m}, \tilde{\boldsymbol{m}})\right. \\
& \left.+w^{-}\left(v^{-}-v_{\text {het }}\right) \Delta Q^{-}(\boldsymbol{m}, \tilde{\boldsymbol{m}})+w_{\text {het }}\left(v^{+}-v^{-}\right) \Delta R(\boldsymbol{m}, \tilde{\boldsymbol{m}})\right)
\end{aligned}
$$


with

$$
\begin{aligned}
\Delta Q^{ \pm}(\boldsymbol{m}, \tilde{\boldsymbol{m}}) & =Q^{ \pm}(\boldsymbol{m}, \tilde{\boldsymbol{m}})-Q^{ \pm}(\boldsymbol{m}, \boldsymbol{m}) \\
& =\frac{1}{4}\left(\tilde{M}_{\mathrm{hom}}^{ \pm}\left(N_{\mathrm{hom}}^{ \pm}-\left(1-\tilde{m}^{\prime}\right) N_{\mathrm{hom}}^{\mp}\right)-M_{\mathrm{hom}}^{ \pm}\left(N_{\mathrm{hom}}^{ \pm}-\left(1-m^{\prime}\right) N_{\mathrm{hom}}^{\mp}\right)\right), \\
\Delta R(\boldsymbol{m}, \tilde{\boldsymbol{m}}) & =R(\boldsymbol{m}, \tilde{\boldsymbol{m}})-R(\boldsymbol{m}, \boldsymbol{m}) \\
& =\frac{1}{8}\left(\tilde{M}_{\mathrm{het}}(1-\tilde{m})\left(N_{\mathrm{hom}}^{+}-N_{\mathrm{hom}}^{-}\right)-M_{\mathrm{het}}(1-m)\left(N_{\mathrm{hom}}^{+}-N_{\mathrm{hom}}^{-}\right)\right) .
\end{aligned}
$$

The activity factors of female mutants, $\tilde{M}_{\text {hom }}^{ \pm}$and $\tilde{M}_{\text {het }}$, must be calculated for rare mutant invaders. Using the condition that the mutant allele does not change the female mating rate we obtain from equation (A2)

$$
\begin{aligned}
\tilde{M}_{\text {hom }}^{ \pm} & =\frac{N_{\text {hom }}^{ \pm}\left(1-m^{\prime}\right) N_{\text {hom }}^{\mp}+(1-m) N_{\text {het }}}{N_{\text {hom }}^{ \pm}\left(1-\tilde{m}^{\prime}\right) N_{\text {hom }}^{\mp}+(1-\tilde{m}) N_{\text {het }}} M_{\text {hom }}^{ \pm} \\
\tilde{M}_{\text {het }} & =\frac{(1-m)\left(N_{\text {hom }}^{+}+N_{\text {hom }}^{-}\right)+N_{\text {het }}}{(1-\tilde{m})\left(N_{\text {hom }}^{+}+N_{\text {hom }}^{-}\right)+N_{\text {het }}} M_{\text {het }} .
\end{aligned}
$$

Note that, in general, $\tilde{M}$ depends on both $\boldsymbol{m}$ and $\tilde{\boldsymbol{m}}$ (e.g., for our model 1; for model 2, the $\boldsymbol{m}$-dependence cancels). Therefore, the activity factor $\tilde{M}$ of invading mutants deviates from the activity factor in a monomorphic mutant population. We can now derive the change of $\Delta Q^{ \pm}$and $\Delta R$ with the mutant variables,

$$
\begin{gathered}
\partial\left(\Delta Q^{ \pm}\right)(\boldsymbol{m}, \tilde{\boldsymbol{m}})=\frac{\tilde{M}_{\text {hom }}^{ \pm}}{4}\left(\frac{N_{\text {hom }}^{ \pm} N_{\text {het }}-\left(1-\tilde{m}^{\prime}\right) N_{\text {hom }}^{\mp} N_{\text {het }}}{N_{\text {hom }}^{ \pm}+(1-\tilde{m}) N_{\text {het }}+\left(1-\tilde{m}^{\prime}\right) N_{\text {hom }}^{\mp}} \partial \tilde{m},\right. \\
\left.\quad+\frac{2 N_{\text {hom }}^{+} N_{\text {hom }}^{-}+(1-\tilde{m}) N_{\text {hom }}^{\mp} N_{\text {het }}}{N_{\text {hom }}^{ \pm}+(1-\tilde{m}) N_{\text {het }}+\left(1-\tilde{m}^{\prime}\right) N_{\text {hom }}^{\mp}} \partial \tilde{m}^{\prime}\right) \\
\partial(\Delta R)(\boldsymbol{m}, \tilde{\boldsymbol{m}})=\frac{\tilde{M}_{\text {het }}}{8} \frac{-\left(N_{\text {hom }}^{+}-N_{\text {hom }}^{-}\right) N_{\text {het }}}{(1-\tilde{m})\left(N_{\text {hom }}^{+}+N_{\text {hom }}^{-}\right)+N_{\text {het }}} \partial \tilde{m} .
\end{gathered}
$$

For a Gaussian mating function with $\left(1-m^{\prime}\right)=(1-m)^{4}$ and $\partial m^{\prime}=4(1-m)^{3} \partial m$ we see from (A33) that $\Delta Q^{ \pm}$is strictly monotonically increasing with increasing choosiness. Since $\Delta Q^{ \pm}(\boldsymbol{m}, \boldsymbol{m})=0$, this implies $\Delta Q^{ \pm} \gtrless 0$ for $\tilde{m} \gtrless m$. Similarly, (A34) implies $\Delta R \gtrless 0$ for $\tilde{m} \gtrless m$ if and only if $N_{\text {hom }}^{+}<N_{\text {hom }}^{-}$, and hence $W_{\text {hom }}^{+}>W_{\text {hom }}^{-}$. If $W_{\text {hom }}^{+}<W_{\text {hom }}^{-}, \Delta R$ has the opposite sign. Note that we reach the same conclusions for many alternative (non-Gaussian) choices of the mating function ( 
possible to allow for different mating preferences of heterozygotes with $(+/+)$ and $(-/-)$ homozygotes.

We now have available all the ingredients to complete our proof. Assume that $W_{\text {hom }}^{ \pm}>0>$ $W_{\text {het }}$ and (without restriction) $W_{\text {hom }}^{+}>W_{\text {hom }}^{-}$. We have shown that in this case $v^{+}>v^{-}>v_{\text {het }}$ and $\Delta Q^{ \pm}, \Delta R>0$ for a modifier that increases female choosiness. Since all elements of the vectors $\boldsymbol{v}$ and $\boldsymbol{w}$ must be positive, equation (A28) implies that the leading eigenvalue of $\boldsymbol{A}_{\boldsymbol{m}, \tilde{\boldsymbol{m}}}$ is positive. Hence, the modifier will invade. Similarly, it follows that $W_{\text {hom }}^{ \pm}<0<W_{\text {het }}$ implies that modifiers for weaker choosiness will invade. Finally, for $W_{\mathrm{hom}}^{+}=W_{\mathrm{hom}}^{-}=W_{\text {het }}$ we find $v^{+}=v^{-}=v_{\text {het }}$ and the invasion fitness of all modifiers vanishes.

These results can be extended to a model in which the ecological and mating locus are linked. We then need to split the heterozygote mutant individuals $\nu_{\text {het }}$ into two classes, $\nu_{\text {het }}^{ \pm}$, depending on whether the mutant allele at the mating locus is associated with the + or the - allele at the ecological locus. Let $\rho>0$ be the recombination rate between the two loci. Then the dynamical equations can again be given in matrix form, $\dot{\boldsymbol{\nu}}=\boldsymbol{A}_{\boldsymbol{m}, \tilde{\boldsymbol{m}}}^{(4)} \boldsymbol{\nu}$, with the 4-dimensional mutant vector $\boldsymbol{\nu}=\left(\nu_{\text {hom }}^{+}, \nu_{\text {het }}^{+}, \nu_{\text {het }}^{-}, \nu_{\text {hom }}^{-}\right)$and transition matrix

$$
\begin{aligned}
& \boldsymbol{A}_{\boldsymbol{m}, \tilde{\boldsymbol{m}}}^{(4)}= \\
& \left(\begin{array}{cccc}
\frac{1}{2} \phi_{\text {hom }}^{+}-d_{\text {hom }}^{+}+Q^{+} & \frac{1-\rho}{2}\left(\phi_{\text {het }}+2 R\right) & \frac{\rho}{2}\left(\phi_{\text {het }}+2 R\right) & 0 \\
\frac{1}{2} \phi_{\text {hom }}^{+}-Q^{+} & \frac{1-\rho}{2}\left(\phi_{\text {het }}-2 R\right)-d_{\text {het }} & \frac{\rho}{2}\left(\phi_{\text {het }}-2 R\right) & 0 \\
0 & \frac{\rho}{2}\left(\phi_{\text {het }}+2 R\right) & \frac{1-\rho}{2}\left(\phi_{\text {het }}+2 R\right)-d_{\text {het }} & \frac{1}{2} \phi_{\text {hom }}^{-}-Q^{-} \\
0 & \frac{\rho}{2}\left(\phi_{\text {het }}-2 R\right) & \frac{1-\rho}{2}\left(\phi_{\text {het }}-2 R\right) & \frac{1}{2} \phi_{\text {hom }}^{-}-d_{\text {hom }}^{-}-Q^{-}
\end{array}\right)
\end{aligned}
$$

with $Q^{ \pm}$and $R$ as defined above. The crucial observation now is that if $\boldsymbol{w}=\left(w^{+}, w_{\text {het }}, w^{-}\right)$ is the dominant right eigenvector of the three-dimensional matrix $\boldsymbol{A}_{\boldsymbol{m}, \tilde{\boldsymbol{m}}}$ as defined in equation (A19), then $\boldsymbol{w}^{(4)}=\left(w^{+}, w_{\text {het }} / 2, w_{\text {het }} / 2, w^{-}\right)$is the dominant eigenvector of the fourdimensional matrix $\boldsymbol{A}_{\boldsymbol{m}, \tilde{\boldsymbol{m}}}^{(4)}$ with the same eigenvalue $\lambda(\boldsymbol{m}, \tilde{\boldsymbol{m}})$. Neither the dominant eigenvalue nor the corresponding eigenvector depend on $\rho$. We thus find that the invasion properties are independent of the recombination rate. This is consistent with similar findings by Matessi et al. (2001) for the symmetric model and weak selection. Consequently, our above results, which were derived for $\rho=0.5$, apply equally for any value of $\rho$. 
Note that the preceding analysis is valid for mating modifiers of arbitrary size. However, only for small modifiers are invading mutants also guaranteed to reach fixation (unless the population is at an evolutionary equilibrium; Geritz et al. 2002; Geritz 2005). Furthermore, at a stable equilibrium with intermediate $m$, the invasion fitness for all modifiers is zero. Since this implies that the second derivative of the invasion fitness in the mutant direction is also zero, this is a non-generic case of an evolutionary singularity according to the classification of Geritz et al. (1998). In particular, polymorphisms of $m$ may be maintained in the vicinity of the singularity, but there is no "evolutionary branching".

\section{Appendix 3: Evolutionary equilibria}

\subsection{Analysis of the symmetric model without sexual selection}

Here we show how to derive the evolutionary equilibrium with respect to female choosiness in the symmetric model without sexual selection. From equation (A9) (and because $\phi_{\text {hom }}=$ $\left.\phi_{\text {het }}\right)$, the equilibrium condition for intermediate levels of choosiness $\left(0<\left\{m, m^{\prime}\right\}<1\right)$, $W_{\text {hom }}=W_{\text {het }}$ (Appendix 2), requires $d_{\text {hom }}=d_{\text {het }}$. Using equation (A8), this latter condition is fulfilled if

$$
n=\hat{n}=\frac{2(c+k-c k)-c^{\prime}}{c-k}
$$

The resulting phenotypic distribution is bimodal (i. e., $n<1)$ if $k<\left(c^{\prime}-c\right) /(3-2 c)$. Note that $\hat{n}$ is the ratio that would be reached in an asexual population of three competing clones with phenotypes $-x, 0$, and $x$. In the sexual case, $n=\hat{n}$ can be reached if assortative mating can evolve. To obtain the equilibrium value for $m$ and $m^{\prime}$, observe first that mean fitness at the equilibrium must be 0 . We thus find $d_{\text {hom }}=1$ and therefore $B_{\text {hom }}=N_{\text {hom }}=N_{\text {hom }} \phi_{\text {hom }}$, using (A10a) and the fact that $\phi_{\text {hom }}=1$. Using equations (A4a) and (A6a), this condition can be expressed as

$$
N_{\text {hom }}\left(\left(m^{\prime}-1\right) M_{\text {hom }}+\frac{n^{2}}{4} M_{\text {het }}\right)=0 .
$$

Plugging in the expression for $M_{\text {hom }}$ and $M_{\text {het }}$ (A5a) and rearranging leads to 


$$
1-m=\frac{\frac{n^{2}}{4}+\left(1-m^{\prime}\right)\left(\frac{n^{2}}{4}-n\right)}{\left(\frac{n^{2}}{4}+1-m^{\prime}\right)\left(1-\frac{n}{2}\right)} .
$$

For $0<n<2$, this condition can be solved numerically (for a given functional relationship between $m$ and $m^{\prime}$ ) to yield the evolutionarily stable value of $m$. Assuming that $m^{\prime}$ is an increasing function of $m$, the left-hand side of equation (A38) decreases with $m$ whereas the right-hand side increases with $m$ (because $n^{2} / 4-n<0$ ). Therefore, there can be only one intersection point, and the solution is always unique. For $\hat{n} \leq 0$ in (A36), $m=m^{\prime}=1$, and for $\hat{n} \geq 2, m^{\prime}=m=0$.

\section{Appendix 3.2: Analysis of the symmetric model with sexual selection}

Our aim here is to derive an analytical expression for $\Delta_{W}=\Delta_{\phi}+\Delta_{d}$ as a function of the mating parameters $m$ and $m^{\prime}$ at the symmetric equilibrium. Using $\phi_{\text {female }}=1$ and $\phi_{\text {male, het }}(n /(2+n))+\phi_{\text {male, hom }}(2 /(2+n))=1$, we obtain

$$
\Delta_{\phi}=\frac{1}{2}\left(\phi_{\text {male, hom }}-\phi_{\text {male, het }}\right)=\frac{2+n}{4}\left(1-\phi_{\text {male, het }}\right)
$$

and with equation (A10)

$$
\Delta_{d}=-\left(d_{\mathrm{hom}}-d_{\mathrm{het}}\right)=\frac{B_{\mathrm{het}}}{N_{\text {het }}}-\frac{B_{\mathrm{hom}}}{N_{\mathrm{hom}}} .
$$

Using equations (A4) and (A6), these are functions of $m, m^{\prime}$, and $n=N_{\text {het }} / N_{\text {hom. We }}$ therefore need a solution for $n$ at the ecological equilibrium. Using $B_{\text {hom }}=N_{\text {hom }} d_{\text {hom }}$ and $B_{\text {het }}=N_{\text {het }} d_{\text {het }}$ (from eq. A10), we find

$$
\begin{aligned}
n & =\frac{d_{\mathrm{hom}} B_{\mathrm{het}}}{d_{\mathrm{het}} B_{\mathrm{hom}}}=\frac{K_{\mathrm{het}}}{K_{\mathrm{hom}}} \cdot \frac{C_{\mathrm{hom}}}{C_{\mathrm{het}}} \cdot \frac{B_{\mathrm{het}}}{B_{\mathrm{hom}}} \\
& =\frac{1}{1-k} \cdot \frac{2-c^{\prime}+(1-c) n}{2-2 c+n} \cdot\left(2-\frac{2 m^{\prime}}{2+(1-m) n+\left(2-m^{\prime}+(1-m) n\right) n / 2}\right) .
\end{aligned}
$$

This is a fourth-order equation in $n$ that can be solved analytically (e.g., by using Mathematica). For most of the parameter space only a single positive solution exists. In some rare cases (in our example with $c^{\prime}=1$ with very low $c$ and high $m$ and $m^{\prime}$ ) there are three positive solutions and the ecological system (A10) is bistable. However, only a single solution for $n$ 
was found at the evolutionary equilibrium for $m$ and $m^{\prime}$ in all cases considered. Evolutionary equilibria at random mating and complete isolation can be found analytically (see below). For equilibria with partial isolation, the condition $\Delta_{W}\left(m, m^{\prime}\right)=0$ is solved numerically for $m$ and $m^{\prime}$.

Sexual selection and mating rates. According to equation (A39), sexual selection is determined by $\phi_{\text {male, het }}$, the mating rate of heterozygous males, which, at the symmetric equilibrium, can be written as

$$
\phi_{\text {male, het }}=\frac{2(1-m)}{2+n(1-m)-m^{\prime}}+\frac{n}{2(1-m)+n} .
$$

Sexual selection disfavors heterozygotes (and, thus, favors homozygotes) if $\phi_{\text {male, het }}<1$, which is the case if equation (17) is true.

Stability of complete isolation. Complete isolation is characterized by $m=m^{\prime}=1$. Note that this does not yet imply $n=0$. For $N_{\text {het }}>0$, we find $B_{\text {hom }}=N_{\text {hom }}+N_{\text {het }} / 4$ and $B_{\text {het }}=N_{\text {het }} / 2$ and obtain an ecological equilibrium with $d_{\text {hom }}=1+n / 4$ and $d_{\text {het }}=1 / 2$. However, since also $\phi_{\text {male, het }}=\phi_{\text {male, hom }}=1$, this implies $\Delta_{d}<0$. The equilibrium is therefore evolutionarily unstable.

For $n=0$, we obtain $d_{\text {hom }}=\phi_{\text {hom }}=1$ and $W_{\text {hom }}=0$. For ecological and evolutionary stability, we need to consider the limit $m, m^{\prime} \rightarrow 1$ and $n \rightarrow 0$. To leading order, we obtain $B_{\text {het }}=2\left(1-m^{\prime}\right) N_{\text {hom }}+N_{\text {het }} / 2$ and thus, from (A10b), that $N_{\text {het }}=0$ is ecologically stable for $m^{\prime} \rightarrow 1$ if and only if $d_{\text {het }}>1 / 2$. Evolutionary stability with respect to the invasion of modifiers for decreased choosiness is given if and only if $W_{\text {het }}=\left(1+\phi_{\text {male, het }}\right) / 2-d_{\text {het }}<0$. For $m^{\prime}$ and $m$ near 1 , (A10b) implies $n=4\left(1-m^{\prime}\right) /\left(2 d_{\text {het }}-1\right)$ at the ecological equilibrium. Using this value for $n$ in (A42), the mating rate of heterozygote males is

$$
\phi_{\text {male, het }}=\lim _{m, m^{\prime} \rightarrow 1} \frac{1-m^{\prime}}{\left(d_{\text {het }}-1 / 2\right)(1-m)+\left(1-m^{\prime}\right)} .
$$

Defining $\lim _{m, m^{\prime} \rightarrow 1}\left(1-m^{\prime}\right) /(1-m)=\kappa$, we obtain the following condition for the evolutionary stability of complete isolation: 


$$
d_{\text {het }}>\frac{1}{2}\left(1-\kappa+\sqrt{\kappa^{2}+2 \kappa}\right) .
$$

With $d_{\text {het }}=(2-2 c) N_{\text {hom }} / K_{0}$ and $N_{\text {hom }} / K_{0}=(1-k) /\left(2-c^{\prime}\right)$ (from $\left.d_{\text {hom }}=1\right)$ we obtain the stability condition (15).

Stability of monomorphic equilibria at the ecological locus. The local stability of a monomorphic equilibrium (say with the ' + ' allele fixed) can be determined analytically by focusing on the fitness of an invading (mutant) '-' allele. As long as this allele is rare, it will occur almost exclusively in heterozygotes. The monomorphic equilibrium is stable if the mutant allele cannot invade, which is the case if $W_{\text {het }}<0$. It is easy to see (from equations A1b and A8 for $N_{\text {hom }}=K_{\text {hom }}$ and $\left.N_{\text {het }} \rightarrow 0\right)$ that $\phi_{\text {male, het }}=1-m$ and $d_{\text {het }}=(1-c)(1-k)$. Together with equation (A9b), this leads to condition (18).

\section{Appendix 4: General non-Gaussian competition functions}

In Figure 4, we analyzed the model for two extreme cases of non-Gaussian competition functions $\left(c^{\prime}=c\right.$ and $\left.c^{\prime}=1\right)$. Here we present a general analysis of what stable evolutionary equilibria are possible in the symmetric model for arbitrary choices of $c$ and $c^{\prime}$.

Model without sexual selection. In the model without sexual selection (Fig. A1a), the complete-isolation equilibrium is stable if condition (14) is true. This is possible only if the numerator on the right-hand side is positive, which is the case if

$$
c^{\prime}>2 c
$$

Furthermore, the partial-isolation regime requires that, for some $k$, neither condition (13) nor (14) is fulfilled. This is possible only if the right-hand side of inequality (13) is greater than the right-hand side of inequality (14), which is the case if

$$
c^{\prime}<4 c-2 c^{2}
$$

Plugging this conditions into inequality (14), we see that, in this case, complete isolation is stable if and only if $c>k$. Vice versa, the bistable $\mathrm{R} / \mathrm{C}$ regime is possible only if $c^{\prime}>4 c-2 c^{2}$. 
Using (13) we see that for $c^{\prime}$ larger than this value, random mating is stable if and only if $k>c$. Thus, the partial-isolation regime can only exist for $k<c$ and the $\mathrm{R} / \mathrm{C}$ regime only for $k>c$, showing that the situation in Figure 4a is generic for the model without sexual selection.

If the competition function is quadratic (as in Matessi et al. 2001) then $c^{\prime}=4 c$ (possible only for $c<1 / 4)$. As $4 c>4 c-2 c^{2}$, the partial-isolation regime is never possible for this function, complete isolation is always locally stable, and random mating can be stable only for $k>c$. It can be shown that these conclusions also hold for the model with sexual selection.

Finally, in the model without sexual selection, there is no $\mathrm{P} / \mathrm{C}$ regime. This is because the equilibrium condition $\Delta W=0$ is never fulfilled for more than one value of $m$ (see Appendix 3.1).

Model with sexual selection. In the model with sexual selection (Fig. A1b), the complete isolation equilibrium is stable if condition (15) is true. This is possible only if the numerator on the right-hand side is positive, which is the case if

$$
c^{\prime}>4 c-2 .
$$

This condition is always fulfilled if the competition function is convex, that is, if $c^{\prime}>2 c$. Furthermore, the partial-isolation regime requires that, for some $k$, neither condition (13) nor (15) is fulfilled, which is possible only if

$$
c^{\prime}<10-4\left(\frac{1}{c}+c\right) .
$$

The reverse condition holds for the $\mathrm{R} / \mathrm{C}$ regime.

The above results are summarized in Figure A1. Several observations are of interest.

Stability of complete isolation is favored by large $c^{\prime}$, but disfavored by large $c$. In particular, without sexual selection, complete isolation can be stable only if the competition function is convex $\left(c^{\prime}>2 c\right)$. (2) For some combinations of $c$ and $c^{\prime}$, either the partial isolation or the R/C regimes are impossible. In particular, the partial-isolation regime does not exist for quadratic competition functions (which is why it was not found by Matessi et al. 2001), whereas the R/C regime does not exist in the absence of sexual selection for Gaussian competition functions 


\section{Without sexual selection}

(a)

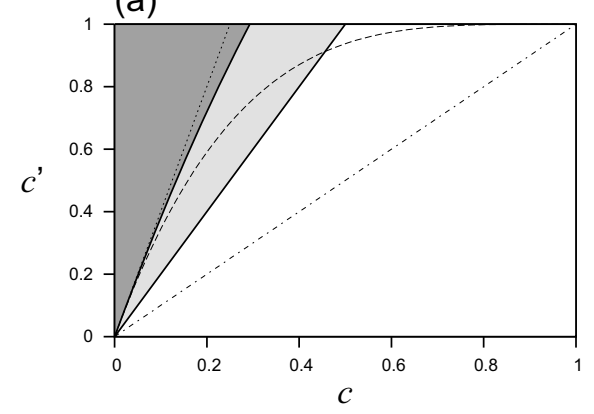

With sexual selection

(b)

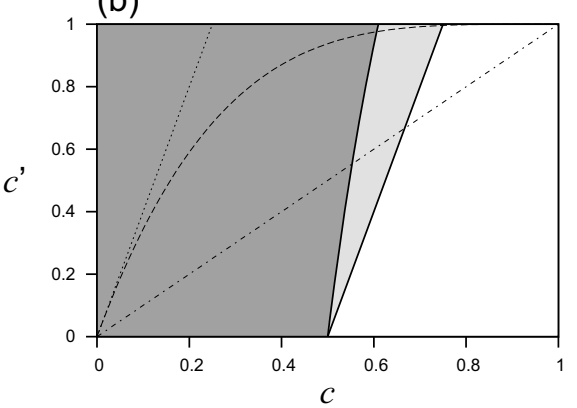

Figure A1: Possible evolutionary equilibria of female choosiness $m$ as a function of the competition parameters $c$ and $c^{\prime}$. The dashed line corresponds to a Gaussian competition function (eq. 2), and the dotted line to a quadratic one (as in Matessi et al. 2001). The dash-dotted line represents the case $c^{\prime}=c$ (see Fig. 4a,c), and the upper boundary of the graph the case $c^{\prime}=1$ (see Fig. 4b,d). For each point, the grayscale indicates which evolutionary equilibria occur for different choices of the parameter $k$ (i. e., along a horizontal line in Fig. 1 and 4). There are three different domains with increasing stability of complete isolation. In the white domain, complete isolation cannot be stable, and the only possible regimes are $\mathrm{R}$ and $\mathrm{P}$. In the light gray domain, complete isolation cannot be stable if random mating is stable, and therefore the bistable $\mathrm{R} / \mathrm{C}$ regime does not exist. For the model without sexual selection, stability of complete isolation further requires disruptive selection $(c>k)$. In the dark gray domain, complete isolation is stable whenever random mating is unstable, and thus the P regime does not exist. Furthermore, in the model without sexual selection, stability of random mating is restricted to $c<k$.

(Fig. 4b, d). (3) For the model without sexual selection, the partial-isolation and $\mathrm{R} / \mathrm{C}$ regimes can only exist for disruptive selection $(c>k)$ and stabilizing selection $(c<k)$, respectively.

\section{Appendix 5: Asymmetric model}

So far, we have assumed a symmetric shape of the carrying capacity $K(X)$ around the heterozygote phenotype at $X=0$. Both homozygote phenotypes thus have the same carrying capacity. As a consequence, the two homozygote classes can be lumped at the symmetric polymorphic equilibrium, which simplifies the analysis. In the following, we relax this assumption. In addition to the parameter $k$ that measures the average strength of stabilizing selection, we introduce an additional asymmetry parameter $\Delta_{k}$ that determines the difference of the selection parameters for opposite homozygotes $k^{+}$and $k^{-}$, 


$$
k^{ \pm}=k\left(1 \pm \Delta_{k}\right)
$$

Similarly, we can also use an asymmetric competition function and define

$$
c^{ \pm}=c\left(1 \pm \Delta_{c}\right)
$$

Our general invasion analysis in Appendix 2 shows that a vanishing invasion fitness is characterized by the (necessary and sufficient) condition $W_{\text {hom }}^{+}=W_{\text {het }}=W_{\text {hom }}^{-}=0$. Below, we will use this condition to derive the boundary lines for the stability of random mating and complete isolation. Also the conditions for stability of the monomorphic equilibria are easily extended. A complication for the asymmetric model arises from the fact that, for intermediate values of female choosiness, a full analytical solution of the population dynamical equations (A10) is no longer possible. We therefore need to rely more extensively on numerical analysis.

The boundary line for the stability of random mating $\left(m=m^{\prime}=0\right)$ can be found from the conditions $d_{\mathrm{hom}}^{ \pm}=d_{\mathrm{het}}=1$ (from A9) and $N_{\text {het }}^{2}=4 N_{\text {hom }}^{+} N_{\text {hom }}^{-}$(from A10). After elimination of $N_{\text {hom }}^{ \pm}$and $N_{\text {het }}$ from this system (using A8), we obtain a lengthy quadratic expression in $k$ that can be solved for arbitrary parameters $c, c^{\prime}, \Delta_{c}$, and $\Delta_{k}$. For simplicity, we focus on the case $\Delta_{c}=0$ and the corresponding solution

$$
k=\frac{c c^{\prime}-\left(4 c-2 c^{2}-c^{\prime}\right)\left(\sqrt{1+\Delta_{k}^{2} c^{\prime}\left(4 c-c^{\prime}\right)}-1\right)}{2 c(2-c)-2 \Delta_{k}^{2}\left(2 c(2-c)-c^{\prime}\right)^{2}} .
$$

For complete isolation $\left(m, m^{\prime} \rightarrow 1, N_{\text {het }}=0\right)$ we can follow the derivation in the symmetric case and obtain $d_{\mathrm{hom}}^{ \pm}=1$ from $B_{\mathrm{hom}}^{ \pm}=N_{\mathrm{hom}}^{ \pm}$. From the definition of the death rates in (A8) we then obtain

$$
d_{\text {het }}=\frac{2 c k}{c^{\prime}} \Delta_{c} \Delta_{k}+\frac{2(1-c)(1-k)}{2-c^{\prime}} .
$$

As in the symmetric case, the condition for stability of complete isolation can be derived from the condition for $W_{\text {het }} \leq 0$ in the limit $N_{\text {het }} \rightarrow 0$. For model 1 , we again find that this is equivalent to $d_{\text {het }} \geq 1$, hence

$$
k \leq \frac{\left(c^{\prime}-2 c\right) c^{\prime}}{2(1-c) c_{4}^{\prime}-2 c \Delta_{c} \Delta_{k}} .
$$


For model 2 and with $\kappa=\lim _{m, m^{\prime} \rightarrow 1}\left[\left(1-m^{\prime}\right) /(1-m)\right]$, we get $2 d_{\text {het }}-1=-\kappa+\sqrt{\kappa^{2}+2 \kappa}$, again as in the symmetric case. With (A52) this translates to

$$
k \leq \frac{\left(2+c^{\prime}-4 c\right) c^{\prime}-\left(2-c^{\prime}\right) c^{\prime} \kappa(\sqrt{1+2 / \kappa}-1)}{4(1-c) c^{\prime}-4 c \Delta_{c} \Delta_{k}}
$$

Finally, we obtain two different stability conditions for the two opposite monomorphic equilibria by substituting $k$ and $c$ in the conditions for the symmetric model by $k^{+}$and $c^{+}$, or by $k^{-}$and $c^{-}$, respectively. For model 1 without sexual selection, monomorphic equilibria are stable if and only if

$$
1-\left(1-c^{ \pm}\right)\left(1-k^{ \pm}\right) \leq 0
$$

This is only possible if either $c^{ \pm} \leq 0$ or $k^{ \pm} \leq 0$ (and hence $K_{\text {hom }}^{ \pm} \geq K_{\text {het }}$, i. e., the resource distribution is no longer stabilizing). For model 2, the condition is (see eq. 18)

$$
\frac{m}{2} \geq c^{ \pm}+k^{ \pm}-c^{ \pm} k^{ \pm}
$$

Figure A2 shows the evolutionary regimes of the model with an asymmetric carrying capacity $\left(\Delta_{k}=1 / 3\right)$, but symmetric competition function. The condition for the stability of random mating is changed only slightly as compared to the symmetric case (eq. A51). The stability condition for complete isolation changes only if also the competition function is asymmetric (eq. A53, A54). Thus, the structure of the five evolutionary regimes appears to be very robust. Marked deviations from the symmetric case appear only with regard to the stability of the ecological equilibria in the model with sexual selection (eq. A56 and numerical results). Not surprisingly, an asymmetric carrying capacity tends to increase the stability of the monomorphic equilibrium containing the fitter type of homozygotes. It also increases the region where a stable polymorphic equilibrium does not exist (hatched area in Fig. A2). Whereas, in the symmetric case, the stable polymorphic equilibrium is always at allele frequency $1 / 2$, in the asymmetric case, it may approach the monomorphic equilibrium for intermediate or large $m$. For some parameter values, no (stable or unstable) polymorphic equilibrium exists. However, non-existence or instability of the polymorphic equilibrium is still largely restricted to parameter combinations with $k \gg c$. 

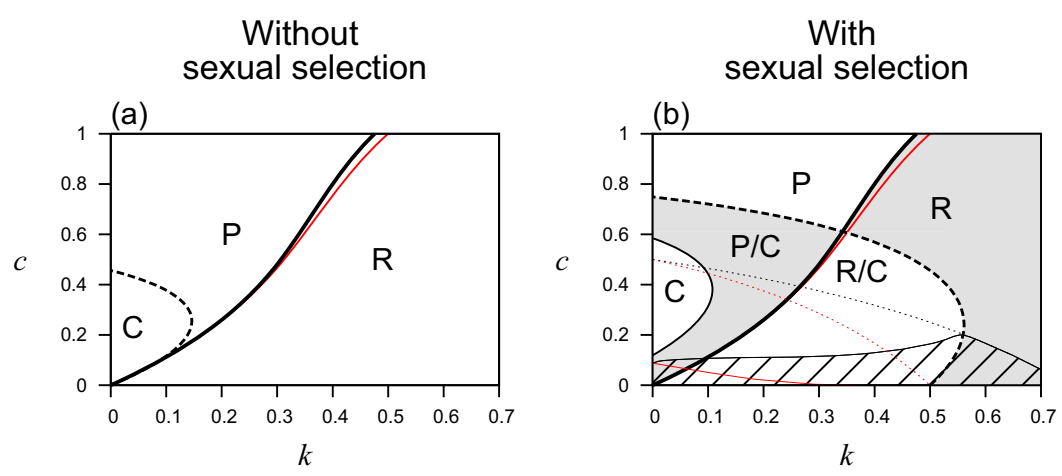

Figure A2: Evolutionary regimes for female choosiness in the model with an asymmetric carrying capacity (see Fig. 1 for details). The asymmetry parameter $\Delta_{k}=1 / 3$ implies that $k^{+}=2 k^{-}$, i.e., stabilizing selection on the $(+/+)$ homozygotes is twice as large as on the $(-/-)$ homozygotes. The thin dotted line (stability of monomorphic equilibrium) refers to the monomorphic equilibrium with the $(+/+)$ homozygotes. The analogous curves for the symmetric model $\left(\Delta_{k}=0\right.$, see Figure 1$)$ are shown in red. Note that the condition for stability of complete isolation (thick dashed line) is independent of $\Delta_{k}$ (see eq. A53 and A54). The boundary of the complete-isolation $(\mathrm{C})$ regime in the model with sexual selection was calculated numerically and, at this resolution, is indistinguishable from the analogous curve for the symmetric model.

\section{References}

Ackermann, M., and M. Doebeli. 2004. Evolution of niche width and adaptive dynamics. Evolution 58:2599-2612.

Arnegard, M. E., and A. S. Kondrashov. 2004. Sympatric speciation by sexual selection alone is unlikely. Evolution 58:222-237.

Bolnick, D. I. 2004a. Can intraspecific competition drive disruptive selection? An experimental test in natural populations of sticklebacks. Evolution 58:608-613.

. 2004b. Waiting for sympatric speciation. Evolution 58:895-899.

. 2006. Multi-species outcomes in a common model of sympatric speciation. Journal of Theoretical Biology 241:734-744.

Bolnick, D. I., R. Svanbäck, J. A. Fordyce, L. H. Yang, J. M. Davis, C. D. Husley, and M. L. Forister. 2003. The ecology of individuals: incidence and implications of individual specialization. American Naturalist 161:1-28.

Bürger, R. 2005. A multilocus analysis of intraspecific competition and stabilizing selection on a quantitative trait. Journal of Mathematical Biology 50:355-396. 
Bürger, R., and K. Schneider. 2006. Intraspecific competitive divergence and convergence under assortative mating. American Naturalist 167:190-205.

Bürger, R., K. Schneider, and M. Willensdorfer. 2006. The conditions for speciation through intraspecific competition. Evolution 60:2185-2206.

Caswell, H. 2000. Matrix population models: construction, analysis and interpretation. 2nd edition. Sinauer, Sunderland.

Christiansen, F. B., and V. Loeschcke. 1980. Evolution and intraspecific exploitative competition. I. One-locus theory for small additive gene effects. Theoretical Population Biology 18:297-313.

Darwin, C. 1859. On the origin of species by means of natural selection or the preservation of favored races in the struggle for life. 1st edition. J. Murray, London.

Dieckmann, U., and M. Doebeli. 1999. On the origin of species by sympatric speciation. Nature 400:354-357.

Dieckmann, U., and R. Law. 1996. The dynamical theory of coevolution: a derivation from stochastic ecological processes. Journal of Mathematical Biology 34:579-612.

Dieckmann, U., M. Doebeli, J. A. J. Metz, and D. Tautz, eds. 2004. Adaptive speciation. Cambridge University Press, Cambridge, UK.

Doebeli, M. 1996. A quantitative genetic competition model for sympatric speciation. Journal of Evolutionary Biology 9:893-909.

Doebeli, M., and U. Dieckmann. 2003. Speciation along environmental gradients. Nature 421:259-264.

2005. Adaptive dynamics as a mathematical tool for studying the ecology of speciation processes. Journal of Evolutionary Biology 18:1194-1200.

Doebeli, M., U. Dieckmann, J. A. J. Metz, and D. Tautz. 2005. What we have also learned: adaptive speciation is theoretically plausible. Evolution 59:691-695.

Doebeli, M., H. J. Blok, O. Leimar, and U. Dieckmann. 2007. Multimodal pattern formation in phenotype distribution of sexual populations. Proceedings of the Royal Society of London B, Biological Sciences 274:347-357. 
Felsenstein, J. 1981. Skepticism towards Santa Rosalia, or why are there so few kinds of animals. Evolution 35:124-138.

Fry, J. D. 2003. Multilocus models of sympatric speciation: Bush versus Rice versus Felsenstein. Evolution 57:1735-1746.

Gavrilets, S. 2004. Fitness landscapes and the origin of species. Princeton University Press, Princeton, NJ.

2005. "Adaptive speciation" - it is not that easy: a reply to Doebeli et al. Evolution 59:696-699.

Geritz, S. A. H. 2005. Resident-invader dynamics and the coexistence of similar strategies. Journal of Mathematical Biology 50:67-82.

Geritz, S. A. H., and E. Kisdi. 2000. Adaptive dynamics in diploid, sexual populations and the evolution of reproductive isolation. Proceedings of the Royal Society of London B, Biological Sciences 267:1671-1678.

Geritz, S. A. H., E. Kisdi, G. Meszéna, and J. A. J. Metz. 1998. Evolutionary singular strategies and the adaptive growth and branching of the evolutionary tree. Evolutionary Ecology 12:35-57.

Geritz, S. A. H., M. Gyllenberg, F. J. A. Jacobs, and K. Parvinen. 2002. Invasion dynamics and attractor inheritance. Journal of Mathematical Biology 44:548-560.

Gíslason, D., M. M. Ferguson, S. Skúlason, and S. S. Snorasson. 1999. Rapid and coupled phenotypic differentiation in Icelandic Arctic char (Salvelinus alpinus). Canadien Journal of Fisheries and Aquatic Sciences 56:2229-2234.

Gourbière, S. 2004. How do natural and sexual selection contribute to sympatric speciation? Journal of Evolutionary Biology 17:1297-1309.

Gyllenberg, M., and G. Meszéna. 2005. On the impossibility of coexistence of infinitely many strategies. Journal of Mathematical Biology 50:133-160.

Higashi, M., G. Takimoto, and N. Yamamura. 1999. Sympatric speciation by sexual selection. Nature 402:523-526. 
Karlin, S., and J. McGregor. 1974. Towards a theory of the evolution of modifier genes. Theoretical Population Biology 5:59-103.

Kirkpatrick, M., and S. L. Nuismer. 2004. Sexual selection can constrain sympatric speciation. Proceedings of the Royal Society of London B, Biological Sciences 271:687-693.

Kisdi, E., and S. A. H. Geritz. 1999. Adaptive dynamics in allele space: evolution of genetic polymorphism by small mutations in a heterogeneous environment. Evolution 53:993-1008.

Knudsen, R., A. Klemetsen, P.-A. Amundsen, and B. Hermansen. 2006. Incipient speciation through niche expansion: an example from the Arctic charr in a subarctic lake. Proceedings of the Royal Society of London B, Biological Sciences 273:2291-2298.

Kopp, M., and J. Hermisson. 2006. The evolution of genetic architecture under frequencydependent disruptive selection. Evolution 60:1537-1550.

MacArthur, R. 1969. Species packing, and what interspecies competition minimizes. Proceedings of the National Academy of Sciences of the USA 64:1369-1371.

. 1972. Niche overlap as a function of environmental variability. Proceedings of the National Academy of Sciences of the USA 69:1109-1113.

Matessi, C., A. Gimelfarb, and S. Gavrilets. 2001. Long-term buildup of reproductive isolation promoted by disruptive selection: how far does it go? Selection 2:41-64.

Metz, H., R. M. Nisbet, and S. A. H. Geritz. 1992. How should we define fitness for general ecological scenarios? Trends in Ecology and Evolution 7:198-202.

Polechová, J., and N. H. Barton. 2005. Speciation through competition: a critical review. Evolution 59:1194-1210.

Rosenzweig, M. L. 1978. Competitive speciation. Biological Journal of the Linnean Society 10:275-289.

Roughgarden, J. 1972. Evolution of niche width. American Naturalist 106:683-718.

Rueffler, C., T. J. M. van Dooren, O. Leimar, and P. A. Abrams. 2006. Disruptive selection and then what? Trends in Ecology and Evolution 21:238-245. 
Sasaki, A. 1997. Clumped distribution by neighborhood competition. Journal of Theoretical Biology 186:304-329.

Sasaki, A., and S. Ellner. 1995. The evolutionarily stable phenotype distribution in a random environment. Evolution 49:337-350.

Savolainen, V., M. C. Anstett, C. Lexer, I. Hutton, J. J. Clarkson, M. V. Norup, M. P. Powell, D. Springate, N. Salamin, and W. J. Baker. 2006. Sympatric speciation in palms on an oceanic island. Nature 441:210-213.

Schliewen, U. K., D. Tautz, and S. Pääbo. 1994. Sympatric speciation suggested by monophyly of crater lake cichlids. Nature 368:629-632.

Schneider, K. 2007. Long-term evolution of polygenic traits under frequency-dependent intraspecific competition. Theoretical Population Biology 71:342-366.

Schneider, K., and R. Bürger. 2005. Does competitive divergence occur if assortative mating is costly? Journal of Evolutionary Biology 19:570-588.

Schoener, T. W. 1965. The evolution of bill size differences among sympatric congeneric species of birds. Evolution 19:2599-2612.

Seger, J. 1985. Intraspecific resource competition as a cause of sympatric speciation. In P. J. Greenwood, H. P. H., and M. Slatkin, eds., Evolution: Essays in Honour of John Maynard Smith, pages 43-53. Cambridge University Press, Cambridge, UK.

Spichtig, M., and T. Kawecki. 2004. The maintenance (or not) of polygenic variation by soft selection in heterogeneous environments. American Naturalist 164:70-84.

Strecker, U. 2006. Genetic differentiation and reproductive isolation in a Cyprinodon fish species flock from Laguna Chichancanab, Mexico. Molecular Phylogenetics and Evolution 39:865-872.

van Doorn, G. S., and U. Dieckmann. 2006. The long-term evolution of multilocus traits under frequency-dependent disruptive selection. Evolution 60:2226-2238.

van Doorn, G. S., U. Dieckmann, and F. J. Weissing. 2004. Sympatric speciation by sexual selection: a critical reevaluation. American Naturalist 163:709-725. 
Waxman, D., and S. Gavrilets. 2005a. 20 questions on adaptive dynamics: a target review. Journal of Evolutionary Biology 18:1139-1154.

- 2005b. Issues of terminology, gradient dynamics and the ease of sympatric speciation in adaptive dynamics. Journal of Evolutionary Biology 18:1214-1219. 